\title{
The Effectiveness of Activation of Prior Mathematical Knowledge During Problem-solving in Physics
}

\author{
Süleyman Turşucu ${ }^{1^{*}}$, Jeroen Spandaw ${ }^{2}$, Marc J. de Vries ${ }^{2}$ \\ ${ }^{1}$ Radboud University Nijmegen, THE NETHERLANDS \\ 2 Delft University of Technology, THE NETHERLANDS
}

Received 19 April 2019 • Revised 14 December 2019 • Accepted 11 January 2020

\begin{abstract}
Mathematics is of major importance in science subjects. Unfortunately, students struggle with applying mathematics in science subjects, especially physics. In this qualitative study we demonstrate that transfer of algebraic skills from mathematics in physics class can be improved by using pre-knowledge effectively. We designed shiftproblems involving instructional models to carry out small interventions in textbook problems. Shift-problems are feasible for teachers to adopt in teaching practice. To gain insight in the extent to which students improved their application of algebraic skills including basic skills and symbol sense behaviour, we selected three grade-10 physics students. In round one, the students solved algebraic physics problems as they appear in physics textbooks. Two weeks later in round two, the same problems were presented as shift problems to them where we activated prior mathematical knowledge by providing systematic rule-based algebraic hints at the start of these tasks. Algebraic skills were presented in a similar way to how these were learned in mathematics textbooks. We observed that students' problem-solving abilities increased from $48.5 \%$ in the first to $81.8 \%$ in the second round, indicating the effectiveness of how we implemented shift-problems. Furthermore, we discussed the implications of our results for the international science audience.
\end{abstract}

Keywords: problem-solving, mathematics education, mathematical knowledge, science education

\section{INTRODUCTION}

Mathematics has a pivotal role in science education (Schoenfeld \& Sloane, 2016; Walshe, Johnston, \& McClelland, 2017), for it offers the tools by which quantitative relationships can be calculated, modelled, represented and predicted (Dierdorp, Bakker, van Maanen, \& Eijkelhof, 2014). On the other hand, science provides rich, relevant and meaningful contexts for mathematics. Despite this close relationship, students in a large number of countries struggle with applying mathematics in science subjects, implying insufficient transfer between these subjects (Jonassen et al., 2017; NCTM, 2013; Redish, 2017; 'TIMMS \& PIRLS', 2018).

Even if students have a solid grasp of mathematics, transfer of this subject to science subjects, especially physics is not assured. One of the very rare studies in this area shows that students with sufficient basic mathematical knowledge enrolled in an algebra-based physics course faced problems with mathematics in physics (Rebello et al., 2007). They concluded that the lack of transfer was not due to students' mathematics knowledge, but inappropriate application of algebra to physics problems.

According to Roorda (2012), the main reason for the lack of transfer is related to compartmentalized thinking in which students see mathematics and science as two different unrelated subjects. This phenomenon is reinforced since in many countries both subjects are taught separately ('Netherlands institute for curriculum development', 2018; Honey, Pearson, \& Schweingruber, 2014; 'TIMMS \& PIRLS', 2019). Indeed, according to Furner and Kumar (2007), offering both subjects separately therefore can be seen as a jigsaw puzzle without any picture. The challenge

(c) 2020 by the authors; licensee Modestum Ltd., UK. This article is an open access article distributed under the terms and conditions of the Creative Commons Attribution License (http://creativecommons.org/licenses/by/4.0/).

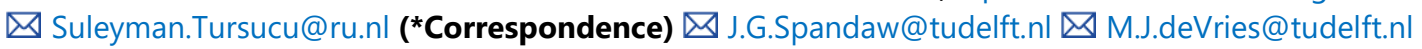




\section{Contribution of this paper to the literature}

- We successfully designed shift-problems to activate prior-mathematical knowledge by providing systematic rule-based systematic hints with emphasis on symbol sense at the start of algebraic physics problems in physics textbooks.

- Through this intervention, students' problem-solving abilities increased from $48.5 \%$ to $81.8 \%$, suggesting high effectiveness of our implementation of shift-problems.

is to integrate the pieces of the puzzle. How this can be done properly, is explained in the sections below. In addition, there can be a mismatch in pedagogical approaches between mathematics and science teachers (Turşucu et al., 2018b), especially in teaching mathematical methods such as algebraic techniques (Drijvers et al., 2011).

A remedy for both compartmentalized thinking and the mismatch above may be coherent mathematics and science education (CMSE) that is of major importance for students (Berlin \& White, 2010, 2012, 2014). The idea behind CMSE is fostering connection between mathematics and science education through, for example alignment of notations. As part of CMSE, the discrepancy in pedagogical approaches may require alignment of teaching approaches, and also improvement of mathematical proficiency (Kilpatrick, Swafford, \& Findell, 2001) that contains the five interwoven strands adaptive reasoning, conceptual understanding, procedural fluency, productive disposition and strategic competence. Concerning algebra education, especially important are the second and third strands. Together, these strands form algebraic expertise (Arcavi, 1994; Andrá et al., 2015) that includes algebraic skills with emphasis on procedural fluency in relation to conceptual understanding. Symbol sense is the part of algebraic skills involving conceptual understanding, relating to "an intuitive feel for when to call on symbols in the process of solving a problem, and conversely, when to abandon a symbolic treatment for better tools" (Arcavi, 1994, p. 25). Moreover, symbol sense contains the ability to choose a sensible systematic problem-solving strategy based on relevant aspects of an algebraic expression.

Through examples, Arcavi (1994) identified eight behaviors of symbol sense that show the intertwinement between conceptual understanding and procedural skills. A key behavior of symbol sense is flexible manipulation skills. While being in control of the work, flexible manipulation skills includes flexible manipulation of expressions (both technical and with insight). Two interconnected concepts characteristics of flexible manipulation skills are having a gestalt view on algebraic expressions and dealing in a suitable manner with their visual salience. The Gestalt gestalt view contains is "the ability to consider an algebraic expression as a whole, to recognize its global characteristics, to 'read through' algebraic expressions and equations, and to foresee the effects of a manipulation strategy" (Bokhove \& Drijvers, 2010, p. 43). Visual salience deals with contains local salience, i.e. sensitivity towards visual cues in local symbols such as exponents, fractions and square root signs, and pattern salience relating to sensitivity towards global patterns in algebraic expressions. In short, both local and pattern salience are part of visual salience; they deal with sensitivity towards local and global cues. This implies that flexible manipulation skills and thus algebraic expertise including basic algebraic skills and symbol sense play a major role in the transfer of mathematics to science subjects, in particular to physics. Even though symbol sense is pivotal in relation to transfer, our extensive literature study including Google Scholar and ProQuest revealed that their relationship has hardly been studied, especially the role of activation of prior mathematical knowledge to improve transfer to physics. In fact, there is no single study examining such relationship with emphasis on activation of pre-knowledge. This makes it worthwhile to investigate this matter.

\section{Research Aim and Central Research Question}

In this study we researched the effectiveness of activation of students' prior mathematical knowledge to solve physics problems in regular physics textbooks (Ottink et al., 2014) for which solution algebraic skills were needed. Students solved these problems during interviews. These problems were presented as shift problems (Palha, Dekker, Gravemeijer, \& van Hout-Wolters, 2013). These are small interventions added to regular textbook tasks that are easily adopted by most students and teachers. Shift-problems contain an instructional model to improve students' learning. In many studies the gap between advice offered in educational research and established classroom practices is too large. Indeed, only very few teachers can incorporate such large changes in their teaching (Leahy \& Wiliam, 2012). As a consequence, such studies often have very limited impact on teaching practice. In this study, the instructional model of shift-problems is used to activate prior mathematical knowledge by providing so called systematic hints at the start of these tasks to improve students' systematic problem-solving abilities, especially symbol sense behavior. Throughout this study we distinguished between two types of hints. In addition to systematic hints we sometimes provided small, neutral hints for those students who got stuck and could not continue or asked for help. Such small hints are referred to simply as hints. Regarding the pedagogy of algebra, we used algebraic skills, in particular algebraic techniques similar to how these were learned in their mathematics textbooks (Reichard et al., 2014). 
These problem-solving activities above are were guided by the central research question "How can activation of prior mathematical knowledge be used effectively to improve students' symbol sense behavior in senior pre-university education when solving algebraic physics problems?". This question was divided in two sub questions: (A) "To what extent do students in upper secondary education demonstrate symbol sense behavior when solving algebraic physics problems that occur in their physics textbooks?", and (B) "To what extent do students in senior pre-university education demonstrate symbol sense behavior when solving the same algebraic physics problems that occur in their physics textbooks after activation of prior mathematical knowledge?".

Earlier studies on algebraic problem-solving in physics have shown (e.g., Turşucu et al., 2017, 2018b) that among students in senior pre-university education grade-10 students encounter the biggest transfer problems. Hence, we selected grade-10 physics students to gain deeper understanding in their algebraic problem-solving skills, especially their basic algebraic skills and symbol sense behavior, and whether and to which extent we could improve their problem-solving skills.

The method to operationalize symbol sense behavior is adopted from (Turşucu et al., 2017). This means that we measured both the extent to which students demonstrated symbol sense behavior and the degree to which they applied basic algebraic skills properly during interviews. Symbol sense behaviour became visible through the application of algebraic techniques during procedures involving basic algebraic skills and having a gestalt view on algebraic expressions and dealing with their visual salience.

For stylistic reasons we sometimes refer to 'conceptual understanding' as 'insightful learning' (Kilpatrick, Swafford, \& Findell, 2001). The terms 'procedural fluency', 'procedural skills' and 'basic algebraic skills' are also used interchangeably.

In this study we do not focus on the meaning or nature of physical concepts. The emphasis is on algebraic skills learned in mathematics class and applied to physics problems.

\section{Educational and Scientific Relevance}

Our literature study also reveals that this is the first time that shift-problems are used outside mathematics education. Therefore, this study may contribute to the evaluation of shift-problems. In addition, the case of students who are successful in mathematics, but struggle with the application of mathematics in physics is highly under examined. This study may provide deeper understanding in why such students struggle with physics problems, thereby providing insight in the underlying mechanisms of how they apply mathematics in physics. Moreover, this study may offer insight in how activating prior mathematical knowledge may be used for the benefit of the international mathematics and science audience, especially curriculum developers, mathematics and physics teachers, mathematics and science teacher educators and textbook publishers who aim to enhance transfer between both subjects and strengthen students' experiencing CME more coherence across these subjects. Concerning the first group, it may provide design principles to connect the mathematics curricula to that of physics through core goals and content standards dealing with, e.g., for example, the same pedagogy of using algebraic skills. Mathematics and physics textbooks may follow identical problem-solving approaches which are followed by individual teachers in mathematics and physics class. For students, this is an appropriate transfer scenario (e.g. Alink, van Asselt, \& den Braber, 2012; Berlin \& White, 2012, 2014). Regarding collaboration, both departments may develop strategies in which algebraic skills are used with emphasis on conceptual understanding. In case of mathematics and science teacher educators, they may use the findings of this study in professional teaching programmes to make teachers aware (Girvan, Conneely, \& Tangney, 2016; Turşucu et al., 2018b) of aspects that influence transfer, i.e. aspects that impede and enhance transfer. Together, the approaches issues above are likely to impede compartmentalized thinking in both subjects and improve students' basic algebraic skills and symbol sense behavior in algebraic physics problems.

\section{BACKGROUND}

\section{CMSE and Transfer in The Classroom}

Connecting mathematics and science subjects is possible through alignment, including, for example concept descriptions and pedagogy of equivalent mathematical approaches (Alink, Asselt, \& Braber, 2012). In addition, there is the organization of the learning process in order to reach an appropriate learning line between these subjects (Alink, Asselt, \& Braber, 2012). This requires that certain mathematical concepts are already taught in mathematics class before these were used in science class. Paying sufficient attention to this connection may improve students' transfer of algebraic skills to physics.

Transfer of learning forms the backbone of the CMSE-approach and refers to the application of initial learning to a new learning situation (Lobato, Rhodehamel, \& Hohensee, 2012). Hence, this type of learning is considered as 
one of the main goals of education (Haskell, 2001; Mestre, 2006). The occurrence of transfer is determined by the expert (teacher) through a correction scheme which is compared to the learners' (student) test answers. So transfer either exists or not, indicating a binary outcome. For instance, the period of a spring-mass system is given by the formula $T=2 \cdot \pi \cdot \sqrt{\frac{m}{c}}$. Solving for $m$ correctly yields $m=\frac{c \cdot T^{2}}{4 \cdot \pi^{2}}$ in the experts' scheme. A different solution is regarded false and implies the absence of transfer (Turşucu et al., 2017). Regarding this study, we elaborate on this matter in the methodology section.

In many countries, CMSE in teaching practice depends on various players such as the curriculum and science teacher educators above (Schmidt, Wang, \& McNight, 2005; Turşucu et al., 2018a). The curricula (intended curriculum) describe the general core goals of education and the specific standards which are tested in national final examinations. Textbooks mediate between these curricula and the actual teaching in classrooms (implemented curriculum) which are closely followed by teachers and their students (Stein \& Smith, 2010). Thus, in the Netherlands the key players are curricula, textbooks and teachers (van Zanten \& van den Heuvel - Panhuizen, 2014). For researchers, the textbooks' central role in teaching practice makes it worthwhile to design shift-problems in which instructional models replace a small part of regular textbooks (Palha, Dekker, Gravemeijer, \& van HoutWolters, 2013). In this study we used this pragmatic approach to design instructional models by means of systematic hints about using algebraic techniques in a similar way to that in mathematics textbooks. By providing systematic hints we aim to activate prior mathematical knowledge and improve both students' procedural fluency and symbol sense behavior during algebraic problem solving in physics and strengthen students experiencing more coherence across these subjects.

\section{Context of The Study}

All authors in this study were affiliated with the mathematics and science teacher education program of Delft University of Technology in the Netherlands. Therefore, they were selected from Dutch secondary schools. For this reason, we briefly sketch the Dutch context in relation to education, especially that of pre-university education. The latter lasts six years and consists of three lower years, i.e. lower pre-university education and three upper years, i.e. senior pre-university education.

The Netherlands is regarded as an advanced industrial country (OECD, 2018) where both mathematics and science education at secondary and college level play an important role in the Dutch political agenda (Ministry of Education, Culture and Science, 2019), implying that government officials and policy makers are paying serious attention to both subjects and their relation. Internationally, Dutch lower and senior pre-university education students score accordingly in assessments of these disciplines, including that of physics (TIMMS \& PIRLS, 2018).

\section{Algebra in Senior Pre-university Mathematics Education}

The algebraic skills in Dutch mathematics curricula deal mainly with algebraic activity, for example algebraic manipulation of formulas and expressions, and patterns of relationships between variables. While a formula refers to an expression containing real measurable quantities such as temperature, an expression can be an abstract algebraic expression with abstract mathematical variables (placeholders), or a formula with physical quantities (Drijvers et al., 2011).

According to mathematics curricula, the whole set of mathematical activities above are part of algebraic skills. The latter is divided into general skills including developing systematic problem-solving strategies with insight, and specific skills dealing with algebraic knowledge and manipulation skills. Thus, specific skills in these curricula are identical to basic algebraic skills. Symbol sense is not explicitly mentioned, but is very close to general skills. The description of general and specific skills together is identical to that of algebraic expertise.

In this study we focus on the application of algebraic techniques which are a key element of algebraic skills, especially in algebraic manipulation of expressions. To solve basic algebraic problems correctly, these techniques should be automated. In this sense, they can be seen as part of the basic algebraic skills machinery. Some wellknown algebraic techniques are 'multiplication of both sides' and 'substitution'. The former multiplies both sides of the equation by the same variable, and the latter replaces single variables in expressions. The spectrum of these techniques is presented and described in detail in the next section.

Mathematics curricula pay little attention to the connection with science subjects, in particular with physics. So, alignment focusing on issues such as equations, formulas and pedagogy of using algebraic techniques are absent in mathematics and physics class. Unfortunately, this also applies for the organization of the learning process in order to reach an appropriate learning line of concepts between mathematics and physics (Alink, Asselt, \& Braber, 2012). 
Algebraic expertise

\begin{tabular}{|ll|}
\hline Basic algebraic skills & Symbol sense \\
- Procedural work & - Strategic work \\
- Local focus & - Global focus \\
- Algebraic calculation & - Algebraic reasoning
\end{tabular}

Figure 1. Algebraic expertise with basic algebraic skills and symbol sense

\section{Algebra in Senior Pre-university Physics Education}

Physics education in the Netherlands starts in grade 8 and corresponds to the second year of lower preuniversity education. Since in the next year students decide whether to choose physics or not, grade 8 is regarded as crucial for potential physics students. Physics is introduced through a strong context-concept approach (Turşucu et al., 2018b) where students start with basic quantities such as force and length. The number of formulas is negligible, so students hardly apply algebraic skills to manipulate formulas. This changes slightly in the next year, but the number of formulas is still very limited and the required level of algebraic skills is low. After the transition to senior pre-university education in grade 10, the level of algebraic skills changes significantly (Turşucu et al., 2017).

Physics formulas are algebraic representations of proportionalities, consisting of two or more variables representing real, measurable quantities. Most formulas express proportionality. They involve only products, quotients and powers, but no sums or differences. (Drijvers et al., 2011). For instance, the formula for kinetic energy $E_{\text {kin }}=\frac{1}{2} \cdot m \cdot\left(v_{\text {final }}\right)^{2}$ is proportional to $\left(v_{\text {final }}\right)^{2}$ and that of an enclosed ideal gas $\frac{P \cdot V}{T}=C$ is inversely proportional to $T$. Although proportionalities are of major importance in physics, it does feature very prominently in mathematics education. This may have consequences for transfer of students' algebraic skills.

The formulas are described in the physics curriculum ("Netherlands institute for curriculum development", 2017). The same applies to the algebraic skills needed to manipulate these formulas. The latter can also be found in BINAS, the Dutch information booklet for natural sciences. Students use it in tests and in the final physics examination.

According to the physics curriculum students should be able to derive formulas, for example the derivation of Keplers' law $G \cdot \frac{M}{4 \cdot \pi^{2}}=\frac{r^{3}}{T^{2}}$, straighten algebraic curves such as the period of a pendulum $T=C \cdot \sqrt{l}$, and conduct dimensional analysis, e.g. for example proving that $[r]$ in the attractive gravitational force $F_{G}=G \cdot \frac{m \cdot M}{r^{2}}$ has unit $m$. Over the last few years, such algebraic physics problems have become more important in the upper secondary education physics program. Moreover, these problems require mathematically correct solutions where algebraic expertise include procedural fluency and sensitivity towards visual salience by means of local salience and pattern salience of algebraic expressions.

\section{Closer Look at Algebraic Expertise}

The spectrum of algebraic expertise extends from basic algebraic skills to symbol sense and is shown in Figure 1 (Arcavi, 1994; Drijvers et al., 2011; Turşucu et al., 2018b). While symbol sense deals with strategic work with a global focus and emphasis on algebraic reasoning, basic algebraic skills concerns procedural work with a local focus and algebraic calculation. Algebraic reasoning deals, among other techniques, with extreme cases and symmetry considerations.

Demonstration of a global focus deals with recognizing patterns in formulas. In this study, strategic work refers to a physics student being in control of her work during algebraic problem solving. The student tries to find a different systematic approach when the initial strategy is insufficient. Our focus is on the intertwinement between local and global, and procedural and strategic work.

A key issue in algebraic problem-solving is how to deal with basic algebraic skills and insight in teaching practice should look like. Should teachers focus on basic skills or on insight, or on some kind of hybrid state? Indeed, these questions are very relevant (Schoenfeld, 2016) and lie at the heart of 'Math Wars', i.e. a long-standing debate about the best way to acquire algebraic expertise. This discussion resulted in clashing ideas about curricula, textbooks and classroom practice. During the past few years there has been a shift towards teaching basic skills and insightful learning through an integrated approach (Rittle-Johnson, Schneider \& Star, 2015; Turşucu et al., 2018a): "Without insight, there is no skill, and without skill, there is no insight" (Drijvers, 2011, p.141), indicating that their 
relationship is bidirectional. We adopt this view on algebraic expertise. For classroom practice, such views on algebraic expertise may have consequences for teaching algebraic skills in both subjects. Mathematics teachers introducing the equation $y=b \cdot x^{2}$ may provide context and meaning by referring to the analogous formula for kinetic energy in physics $E_{\text {kin }}=\frac{1}{2} \cdot m \cdot v^{2}$. Physics teachers may do the opposite, which can be considered as activation of prior mathematical knowledge. Earlier studies on learning and instruction have shown that activation of pre-knowledge may contribute to better students' achievements (e.g. Hailikari, Katajavuori, \& Lindblom-Ylanne, 2008; Roorda, 2012). When solving for $v$ in $E_{\text {kin }}=\frac{1}{2} \cdot m \cdot v^{2}$, physics teachers should use the same pedagogical approach taught in math class, i.e. when solving for $x$ in $y=b \cdot x^{2}$. While students should be skillful in basic operations such as isolation of $x^{2}$ and $v^{2}$, they should also understand and be able to explain the mathematics behind these operations.

\section{Pedagogical Approach to Systematic Problem Solving in Physics}

This study distinguishes between using 'ad hoc strategies' and applying 'systematic algebraic strategies' that includes applying algebraic skills with insight as described in the mathematics curriculum ("Netherlands institute for curriculum development", 2018).

For stylistic reasons we use 'systematic algebraic strategies' and 'systematic algebraic approach' interchangeably to denote the same. This also holds for 'ad hoc strategies' and 'ad hoc approach. A 'systematic algebraic approach' refers to a "rule-based problem-solving approach in which algebraic techniques are used with insight, where 'rule' refers to the standard rules for multiplication and division of powers, such as $x^{a} \cdot x^{b}=x^{a+b}$, which play the role of algebraic axioms in high school algebra" (Turşucu et al., 2018b, p. 5). With 'ad hoc strategies' we refer to "mathematical strategies that are not based on standard algebraic rules with insight, and only work for a specific case that may lead to fragmented knowledge, impeding generalization of algebra". Even though such strategies may be useful as initial steps or even lead to correct solutions (Roorda, 2012), students can get stuck in more sophisticated problems requiring insight. Moreover, students may become dependent on an authority such as a teacher telling them whether an approach is algebraically correct or not. Therefore, ad hoc approaches may be harmful for students' transfer of mathematics in physics, mainly because students lack insight into algebraic skills. Other examples of ad hoc strategies are guessing a solution for a problem and then working backwards or the application of ill-understood mnemonics such as the 'formula triangle'. In this study, applying algebraic skills with insight becomes visible during problem-solving in physics. The student applies algebraic techniques during basic algebraic skills procedures and shows sensitivity towards local salience and pattern salience in formulas.

\section{METHODOLOGY}

\section{Selection Criteria for Students}

In this explorative qualitative study with a quantitative component we aimed to gain deeper understanding in students' symbol sense behavior, and to investigate whether shift-problems designed to activate prior mathematical knowledge can improve students' problem-solving in physics for which solution algebraic skills are needed. Beyond qualitative explanations, the quantitative component offers insight into the extent to which students correctly applied systematic algebraic strategies that became visible through the application of basic algebraic skills and symbol sense behaviour. Therefore, this component should not be confused with quantitative studies generalizing results from a larger sample population (Bryman, 2015).

We used convenience sampling (Bryman, 2015) to find one physics teacher together with three students who were available and willing to participate in this study. Thereafter, we used the 'Physics Teachers' Interview Protocol' that is presented in the first section of the Appendix to interview the teacher and select appropriate physics students. During the pilot-phase prior to this study, this protocol was tested on different teachers and social scientists and redesigned several times until the authors agreed on appropriate length and clearness. Selection of students were based on a sufficient mathematics grade and an insufficient physics grade for which the physics teacher used 'Magister', a secondary education student monitoring system ('accounts.magister.net', 2018). According to the Dutch ten-point grading system, a student' grade is insufficient if her grade is less than 5.5. This indicates that students' insufficient physics grade is not due to her insufficient mathematics knowledge, but related to the inadequate application step of mathematics in physics. We also took into account that the students should have a similar knowledge domain at the start of these interviews. Otherwise, it would not have been legitimate to compare their individual performances. Because of these strong selection criteria, we only found one school satisfying them that yielded the anonymized grade-10 physics students Clare, Mary and Sam (the only male student in this study). All these students took mathematics classes and used the mathematics textbook Getal en Ruimte (meaning: Number and Space) (Reichard et al., 2014) and the physics textbook Natuurkunde Overal (meaning: Physics 
Table 1. Students'sufficient mathematics and insufficient physics grades

\begin{tabular}{cccc}
\hline & Clare & Mary & Sam \\
\hline Grade physics & 5.0 & 4.8 & 5.9 \\
\hline Grade mathematics & 6.9 & 6.8 & 7.9 \\
\hline
\end{tabular}

Everywhere) (Sonneveld et al., 2014). Furthermore, as can be seen in Table 1, their mathematics grade is ranging from 6.8 up to 7.9 and their physics grade from 4.8 up to 5.9. Although Sam's 5.9 did not perfectly meet our requirement, we selected this student. In the last section we elaborate on this matter and legitimize our choice.

\section{Design of Shift-problems}

As mentioned above, the students were asked to solve the tasks about algebraic physics problems in two rounds: first, as they appeared in the physics textbook (Ottink et al., 2014), and after two weeks in the second round as shift problems (Palha, Dekker, Gravemeijer, \& van Hout-Wolters, 2013). The design of shift problems is based on the iterative 3D-principle in which the interaction between, in subsequent order, the teacher (D1), the resources (textbooks) (D2) and the students (D3) is described. D1 starts with a learning objective of the teacher, i.e. in this study the improvement of symbol sense behavior, especially the transfer of algebraic skills including algebraic techniques from mathematics to physics for solving algebraic physics problems. Therefore, the tasks should trigger students problem-solving and provide insight in their algebraic expertise with basic algebraic skills and symbol sense behavior. Based on these criteria, in D2 we selected the algebraic physics problems 'Task 1: Specific heat capacity', 'Task 2: Thermal resistance' and 'Task 3: Charged particles' from the physics textbook SysNat (Ottink et al., 2014). These tasks are described in symbolic representations (Goldin, 2000) and focus on algebraic manipulations. During a pilot-phase these problems were solved by other grade- 10 students who did not participate in this study. This offered us information about clearness, length and usability, i.e. that these tasks were doable by students. Afterwards, some of the tasks were adjusted considering these issues. This resulted in 'The Tasks' containing the three tasks above which are presented in the second subsection of Appendix. Next, we imagined a hypothetical learning trajectory (Arthur Bakker, 2004) by predicting how students will react when solving these problems. For instance, we expected students to apply the ad hoc strategies. In D3, they were videotaped while solving these tasks thinking aloud (Charters, 2003).

As to D1' where the apostrophe refers to the second cycle of the 3D-principle, students' work of D3 was analyzed to add refinements to the tasks. We also used insights from the study in which we examined symbol sense behavior (Turşucu et al., 2018b). Pivotal was that students should avoid the application of transfer impeding ad hoc approaches. Instead, these refinements should focus and trigger the application of systematic algebraic strategies with insight. Next, we examined how algebraic techniques are used in the mathematics textbook Getal en Ruimte (Reichard et al., 2014) to provide similar pedagogical approaches to students that they learned in their mathematics textbook. In D2' these principles were used to design algebraic systematic hints for the tasks aiming at activation of prior mathematical knowledge that we refer to as 'activation Hint'. We also designed a 'strategic hint' providing information about how to start a task. The 'activation hint' together with the 'strategic hint' we called systematic hints. The tasks as they appeared in the physics textbook including these systematic hints form shift-problems.

Immediately before starting with shift-problems, students were introduced by an exemplary task explaining how systematic hints are used. Both the introductory task and shift problems are presented in the fourth subsection 'Shift-problems' of Appendix. The systematic hints are summarized below.

Concerning 'strategic hints' we demonstrated through a systematic algebraic approach how to go from $y=a b$ to $a=\frac{y}{b}$ in task 1 , and from $y=\frac{a}{\frac{c}{d}}$ to $y=\frac{a d}{c}$ in task $2 \mathrm{a}$; that if $y=a b$ and $z=a b$, then $y=z$ for task $2 \mathrm{~b}$, and how to go from $y=\frac{a}{b}$ to $a=y b$ for task 3. Regarding 'activation hints' we asked first to solve for $c$, then to apply the [ ]operator that is used to denote the units of quantities. So the [ ]-operator is applied to $c$, i.e. replacing $c$ by [c] and the other quantities by their units for task 1 ; analogously application of the [ ]-operator to $R_{\text {therm }}$ in task $2 \mathrm{a}$; to rewrite $R_{\text {therm }}$ to $1 / R_{\text {therm }}$ for task $2 \mathrm{~b}$, and to first to solve for $f$ and then to apply the [ ]-operator to $f$. We note that the []-operator refers to the unit-operator that is used for dimensional analysis. Moreover, Dutch physics textbooks use this type of notation.

In D3', two weeks after D3, again while being videotaped students solved these shift-problems after being introduced to these tasks by an example explaining how systematic hints are applied. Their work in both D3 and D3' was analyzed with regard to symbol sense behavior and how they used algebraic techniques. To distinguish between D3 and D3' we refer to D3 as the 'first round' and to D3' as the 'second round'. In short, during the first round the tasks were presented as they appeared in the physics textbook without guidance. Next, in the second round we offered a 'strategic hint' for how to start with the task, and an 'activation hint' to activate prior 
Table 2. Coding scheme to analyze students' basic algebraic skills and symbol sense behaviour during algebraic problem-solving in round 1 and 2

\begin{tabular}{ccccc}
\hline Time & Task & Alegbraic techniques & Ad hoc strategies & Trigger \\
\hline$\ldots$ & 1 & $\ldots$ & $\ldots$ & $\ldots$ \\
\hline$\ldots$ & 2 & $\ldots$ & $\ldots$ & $\ldots$ \\
\hline$\ldots$ & 3 & $\ldots$ & $\ldots$ & $\ldots$ \\
\hline
\end{tabular}

mathematical knowledge inn the sense that algebraic techniques are applied in a similar way as in their mathematics textbook.

\section{Design of Students' Task-based Interviews (TBIs)}

The algebraic problem-solving activities in this study took place during interviews conducted by an independent researcher. Such interviews guided by a protocol are called task-based interviews (TBI's) (Maher \& Sigley, 2014). In this study, the TBI's were designed in such a way that the students only interacted with the tasks and the interviewer. Therefore, they were conducted by the independent researcher in an appropriate, quiet place. Each of these TBI's lasted approximately half an hour. The interview protocol consisted of two parts. During the design process in the pilot phase, we considered that the instructions were clear and that the non-participating students above could easily work with it. Based on their feedback, some parts of this protocol were adjusted, resulting in the 'Interview Protocol Physics Students' that is displayed in the fifth subsection of Appendix. As can be seen, in the first part of our protocol students were asked questions about their background, the textbooks they used, and their mathematics and physics grades were double checked. In the second part they were guided by the protocol to solve 'The Tasks'. We note that the same protocol was also used to solve shift-problems. The interviewer only interrupted when the student remained silent for one minute or a procedure or reasoning was not clear enough. Stimulated recall techniques (Geiger, Muir, \& Lamb, 2016) were used to get as much information as possible on the students' work. For those who got stuck and could not continue or asked for help, we provided small neutral hints.

In addition to these qualitative methods above, we measured levels of basic algebraic skills and symbol sense behavior to compare students' individual performance. This will be discussed in the following subsections.

\section{Data Analysis}

\section{Analysis of videotaped data}

We used the seven phases of Powell, Francisco \& Maher (2003) to analyze videotaped data that do not require a rigid order. In 'phase 1 ' we obtained a first and general understanding of how students solved the tasks. Next, interesting and relevant findings were identified. The description of the videotaped data in 'Phase 2' was less important, since detailed information about the audio part of videotaped data was already transcribed ad verbatim in 'Phase 4', for which the students gave consent. In 'Phase 3' we identified critical events such as ad hoc strategies, the application of algebraic techniques and other relevant information during problem-solving requiring mathematical motivation. These are described in 'phase 5 ' where we operationalized our central research question using the coding scheme (spread sheet) in Table 2.

The coding process was based on the students' written solution set to both 'The Tasks' and 'Shift-problems', the transcripts of the audio part of videotaped data and to a lesser extent on the analysis of physical episodes. Students' written work of round one and two were compared to the 'Systematic Solution Set' (solution set in short) in the third subsection of the Appendix and coded using Table 2. This means that students' work was assigned to scores to gain insight in their algebraic problem-solving skills for each round and to determine to which extent shiftproblems improved their symbol sense behavior. For instance, to solve 'Task 1: specific heat capacity' in Appendix systematically and with insight, the first procedure requires division of both sides of $Q=m \cdot[c] \cdot \Delta T$ by $m$, yielding $\frac{Q}{m}=[c] \cdot \Delta T$. Since $\frac{m \cdot[c] \cdot \Delta T}{m}$ is equivalent to $[c] \cdot \Delta T \cdot m \cdot m^{-1}$, this procedure may require sensitivity towards the exponent -1 in $m^{-1}$ and is associated with the symbol sense type local salience. Students may use other strategies, e.g. for example cancellation of the masses in $\frac{m \cdot[c] \cdot \Delta T}{m}$. Such correct mathematical procedures do not affect scores, neither does interchanging procedures.

\section{The coding process}

The observation of critical events during coding was reported in the first column 'Time' of Table 2. The entries of 'Task' represent the tasks in both the first and the second round. Contrary to the columns 'Time', 'Task' and 'Ad 
hoc strategies', the remaining columns consist of drop-down boxes, each having different options. Concerning 'Algebraic Techniques', students' algebraic technique during a procedure anchored the selection (coding) of one of the options 'multiplication of both sides', 'division of both sides', 'substitution' and 'inverting both sides' in the spreadsheet. If the student used ad hoc approaches instead of systematic algebraic strategies, the details were described in 'Ad hoc strategies'. The column 'Trigger' involved the options 'positive', 'negative' or 'missed opportunity'. For appropriate procedures, this was coded either 'positive' with score ' 1 ' indicating a flawless procedure, or an incorrect 'negative' procedure with score ' $0.5^{\prime}$. The neutral student who used ad hoc strategies or overlooked a required procedure, corresponds to the third scenario 'Missed opportunity' with score ' 0 '. As a result, these scores led to SSB (\%) per task. In addition, the overall symbol sense behavior percentage for all tasks, i.e. $\operatorname{OSSB}(\%)=\frac{\text { sum of all task scores }}{11} \cdot 100 \%$, and OBAS $(\%)=\frac{\text { sum of all task scores requiring BAS only }}{4} \cdot 100 \%$, i.e. the overall basic algebraic skills percentage for all tasks were calculated. Each of the numbers ' 11 ' in OSSB (\%) and ' 5 ' in OBAS (\%) are the sum of respectively eleven procedures involving local salience, pattern salience, and basic algebraic skills, and 'five' basic algebraic skills procedures. This implies that a flawless performance in both cases yields the maximum score of 11 for OSSB (\%) and the maximum score of 5 for OBAS (\%).

The acronyms above, denoting certain procedures during algebraic problem-solving, are widely used throughout this manuscript. For clarity we present them below. These are:

- BAS: basic algebraic skills;

- OBAS (\%): the overall basic algebraic skills percentage for the whole set of tasks;

- SSB (\%): the symbol sense behavior percentage per task, and

- OSSB (\%): the overall symbol sense behavior percentage for the whole set of tasks.

Furthermore, a student was considered procedurally fluent when OBAS (\%) $\geq 75 \%$ (3 out of 4 points). OSSB (\%) was sufficient if OSSB (\%) $\geq 72.7 \%$ (8 out of 11 points). These criteria were the result of consensus among the authors after a series of discussions. The norm for a solid domain of basic algebraic skills was agreed on 3 out of 4 points. As to symbol sense behavior, this extends basic algebraic skills, since it also requires insight in algebraic skills. Thus, we took 8 out of 11 points, i.e. a minimum OSSB (\%) score of $72.7 \%$.

\section{Towards Common Findings}

Regarding 'phase 6 ' (constructing storyline), in subsequent order we identified the ad hoc strategies in both rounds, students' SSB (\%) per task, OBAS (\%) and finally OSSB (\%). Next, during 'phase 7' (composing narrative), the findings from 'phase 6 ', students' written solution set and the transcripts were merged into common findings.

With respect to improvement of reliability (Bryman, 2015), the first and second author independently carried out the coding process. This means that first each author independently compared students' written work to the 'Systematic Solution Set' in Appendix and assigned to scores using Table 2. When crosschecking their results, they found that their findings overlapped for approximately $80 \%$. Next, they discussed the remaining $20 \%$ and made adjustments in those areas of their findings. For instance, they agreed on assessing the work of some students who did not work out a certain procedure involving basic algebraic skills or symbol sense behaviour on paper, but immediately wrote down the correct answer. During the interviews with students thinking aloud, they correctly mentioned the required procedure. Therefore, such procedures were considered positive and assigned the score ' 1 ', indicating a flawless procedure. In addition, sometimes the first or second author accidently assigned incorrect scores for a certain procedure. Such issues were thoroughly double checked and discussed by the first and second author, which led to $100 \%$ agreement among them.

\section{RESULTS}

Recall that we distinguish between 'systematic hints' on the one hand and 'hints' on the other hand, which are small, neutral hints for students who got stuck and could not continue or asked for help.

Furthermore, we especially examined tasks for which students maximized their score to $100 \%$ in the second round using systematic hints. In case of two or more of such maximum scores, we only discussed the task that provided the most relevant information.

\section{Clare}

Clare is a female student with a 5.0 for mathematics and a 6.9 for physics. In round one, she faced serious difficulties with task $2 b$ and 3 where she used the ad hoc approach swapping strategy. She only used one hint for task 1 . In round two, Clare did not use any hints. 
Table 3. Clare's basic algebraic skills and symbol sense behavior characteristics during algebraic problem-solving in round 1 and $\underline{2}$

\begin{tabular}{|c|c|c|c|c|c|c|c|c|}
\hline & 1 & $2 a$ & $2 \mathbf{b}$ & 3 & $1^{\prime}$ & $2 a^{\prime}$ & $2 \mathbf{b}^{\prime}$ & $3^{\prime}$ \\
\hline Missed opportunity & 2 & - & 2 & 3 & 2 & - & 1 & - \\
\hline Negative score & - & 0.5 & - & - & - & - & - & - \\
\hline Positive score & 1 & 1 & - & 1 & 1 & 2 & 1 & 4 \\
\hline Task max. score & 3 & 2 & 2 & 4 & 3 & 2 & 2 & 4 \\
\hline SSB (\%) & 33.3 & 75.0 & 0 & 25.0 & 33.3 & 100.0 & 50.0 & 100.0 \\
\hline
\end{tabular}

Table 4. Clare's solutions for task 3 during algebraic problem-solving in round 1 and 2 Round 1: SSB (\%) $=\mathbf{2 5 . 0} \%$ Round 2: SSB (\%) $=100.0 \%$

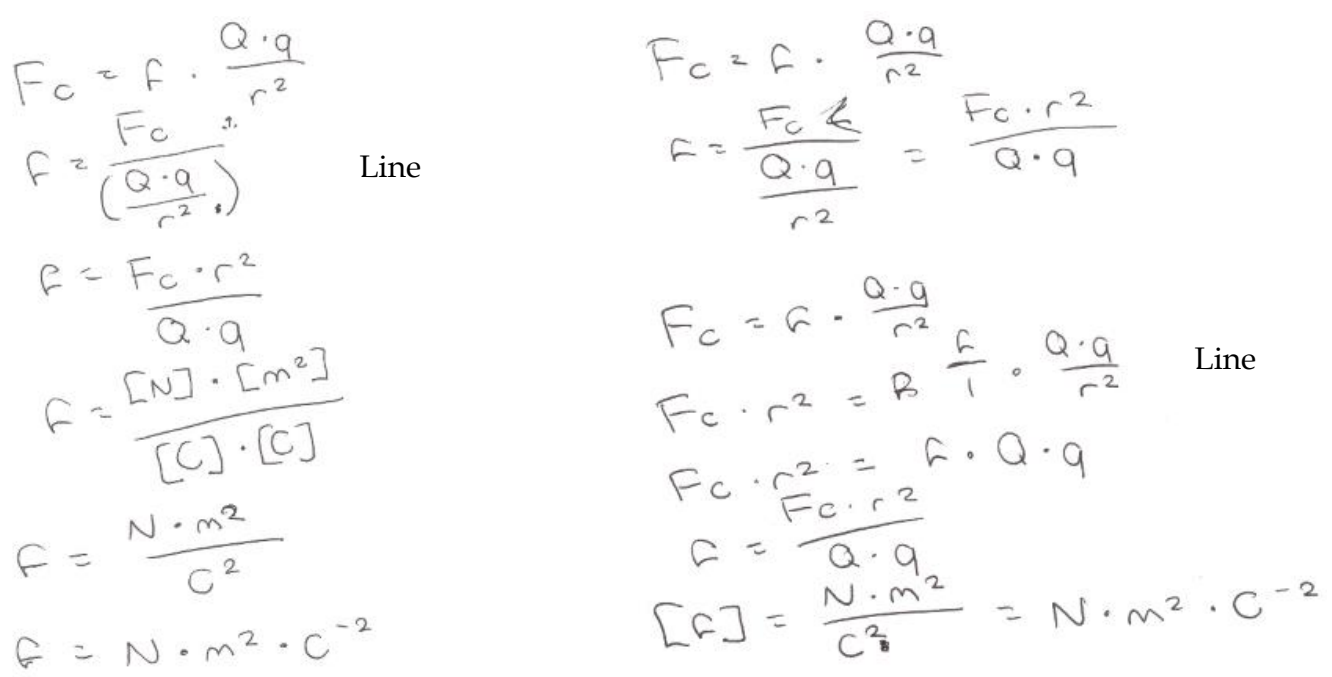

In Table 3, we show her symbol sense behavior characteristics for both rounds. The second (1) up to the fifth column (3) of the first row each represent a task in round one; the sixth (1') up to the ninth column ( $\left.3^{\prime}\right)$ of the first row each represent a task in round two. To distinguish between both rounds, in round two we used apostrophes next to the tasknumbers. 'Missed opportunity', the first column of the second row demonstrates the sum of how many procedures per task she used ad hoc strategies (and) or made no attempt to solve the task.

The entry 'Task max. score', i.e. the fifth row of the first column gives the maximum score of a task. For instance, regarding task 2a in round one, Clare's 'Missed opportunity' equals 0 ; she has one correct procedure with positive score ' 1 ' and one that is incorrect with negative score ' 0.5 '. Overall, her total number of 'Missed opportunity' for round one is seven and consists of five overlooked procedures (four local salience and one pattern salience procedure). In the second round this number is reduced to three including two local salience and one pattern salience procedure. For many overlooked procedures she performs algebraic manipulation 'by head' without writing them on paper. This makes it very difficult to decipher the underlying mechanisms of such procedures. Despite this, we calculated the percentage of her total number of 'Missed opportunity' in round one corresponding to $63.6 \%$ of the perfect score. For round two, this number was $27.3 \%$, implying that Clare significantly reduced the number of overlooked procedures in round two without using ad hoc approaches. Her lowest SSB (\%) is $0 \%$ for task $2 \mathrm{~b}$ and $25.0 \%$ for tasks 3 in round one, and $33.3 \%$ for tasks $1^{\prime}$ in round two. Her highest SSB (\%) corresponds to $75.0 \%$ for task $2 \mathrm{a}$ in round one, and to $100 \%$ for both tasks $2 \mathrm{a}^{\prime}$ and $3^{\prime}$ in round two. For the latter two tasks, Clare used flawless procedures involving basic algebraic skills and local salience procedures. Her OBAS (\%) improved from $\frac{3.5}{4} \cdot 100 \%=87.5 \%$ in the first round to $100.0 \%$ in the second round. Thus, her OBAS (\%) is sufficient. Conerning her OSSB (\%) over both rounds, this improved from $\frac{3.5}{11} \cdot 100 \%=31.8 \%$ to $72.7 \%$. The latter implies that Clare's symbol sense behavior is sufficient. Indeed, she nicely adopted the provided systematic hints for both tasks $2 a^{\prime}$ and $3^{\prime}$, and demonstrated sensitivity towards local salience and pattern salience procedures. Furthermore, even though she struggled with task $2 b$ that only contains pattern salience procedures, her score slightly increased from $0 \%$ to $50 \%$.

For task 3, Clare maximized her SSB (\%) and went from $25 \%$ to $100 \%$ in the second round. This is depicted in Table 4 that gives insight in how she applied the swapping strategy in the first round. In line 2 Clare swaps the whole term $\frac{Q \cdot q}{r^{2}}$ in the denominator of $f=\frac{F_{C}}{\frac{Q \cdot q}{r^{2}}}$ into $f=\frac{F_{C} \cdot r^{2}}{Q \cdot q}$, but does not understand why this step is mathematically 
Table 5. Mary's basic algebraic skills and symbol sense behavior characteristics per task during algebraic problem-solving in round 1 and 2

\begin{tabular}{cccccccc}
\hline & $\mathbf{1}$ & $\mathbf{2 a}$ & $\mathbf{2 b}$ & $\mathbf{3}$ & $\mathbf{1}^{\prime}$ & $\mathbf{2 a}^{\prime}$ & $\mathbf{2} \mathbf{b}^{\prime}$ \\
\hline Missed opportunity & 2 & - & 2 & 3 & - & - & - \\
\hline Negative score & - & - & - & - & - & - & - \\
\hline Positive score & 1 & 2 & - & 1 & 3 & 2 & 2 \\
\hline Task max. Score & 3 & 2 & 2 & 4 & 3 & 2 & 1 \\
\hline SSB (\%) & 33.3 & 100.0 & 0 & 25.0 & 100.0 & 100.0 & 100.0 \\
\hline
\end{tabular}

legitimate. Even though her solution for this problem yields the correct answer, Clare's procedure lacks a rulebased problem-solving approach in which algebraic techniques are used with insight. Remarkably, she used the same approach for task $2 \mathrm{~b}$ where she mentioned: "Bottom one goes to top, middle one remains". It turned out that her mathematics teacher taught her how to swap such terms. In the second round, again after swapping $\frac{Q \cdot q}{r^{2}}$ in $f=\frac{F_{C}}{\frac{Q \cdot q}{r^{2}}}$ she writes $f=\frac{F_{C} \cdot r^{2}}{Q \cdot q}$. Next, Clare paused for longer than one minute after which the interviewer interrupted. Then, as can be seen in line $4^{\prime}$, she decides to multiply both sides with $r^{2}$. She explains that both sides of the equals sign should be divided by $Q \cdot q$ yielding $f=\frac{F_{c} \cdot r^{2}}{Q \cdot q}$. Clare notices that she got the same answer as in the begin of round 2, but that the swapping approach was much easier to use: "I also divided here by swapping this term and that was much easier for me. I did not really used these [systematic] hints. With these [systematic] hints, it takes much more time to solve this question". Nevertheless, she correctly adopted the systematic hints of task 3.

Overall, by adopting the systematic hints in the second round in an appropriate way, Clare converted her lower scores for the high number of 'Missed opportunity' into positive scores. The []-operator was applied flawlessly in all questions. She mentioned that the 'activation hints' of task 2a and 3 were very useful. To a less extent this also holds for task 1 . Despite this, she mentioned that 'her way' of problem-solving including the swapping strategy and algebraic manipulation by memory was easier and quicker to perform. However, Clare did not understand that the systematic hints she applied in the second round helped her to prevent making errors and finishing the problems succesfully. This may change when Clare becomes aware of the benefits of such systematic hints, for example when algebraic manipulations in the next years of senior pre-university education become more difficult. Thus, it is worthwhile to show and make her aware of the importance and effectiveness of the application of systematic hints.

\section{Mary}

Mary is a female student with a 4.8 for mathematics and a 6.8 for physics. In round one, she struggled with task 1 and 3 for which she used the numbering strategy. She only used a hint for task 2a. In round two, Mary did not ask for hints.

Her symbol sense behavior characteristics for both rounds are displayed in Table 5. Mary's total number of 'Missed opportunity' in the first round was seven including two overlooked pattern salience procedures and five local salience procedures for which she used the numbering strategy. In round two, she reduced this total number to three, by applying the numbering strategy for local salience procedures three times. Because she reduced the number of ad hoc approaches and even did not overlook procedures in round two, the percentage of her total number of 'Missed opportunity' dropped from $63.6 \%$ of the perfect score for the first round to $27.3 \%$ for the second. These scores are quite similar to that of Clare. Mary's lowest SSB (\%) is $0 \%$ for task $2 \mathrm{~b}$ and 3 in round one (identical to Clare), and highest for task 2a. While her score for task 3 remained the same in round 2, task $2 \mathrm{~b}$ and 3 increased to the maximum score where she adopted the systematic hints appropriately. For task 3 and $3^{\prime}$ she used the numbering strategy for identical procedures, remarkably avoiding all systematic hints. Mary's OBAS (\%) was already $100.0 \%$ in the first round, and did not change in the second. Therefore, she has a perfect grasp of basic algebraic skills. Her OSSB (\%) improved from $36.4 \%$ to $72.7 \%$ in the second round. Mary's similar scores for the tasks in round two yielded the same OSSB (\%) as Clare. Based on this result, Mary has sufficient symbol sense behavior. Her OSSB (\%) would have even been higher if she had avoided the application of ad hoc strategies in task 3'.

In Table 6, we depicted Mary's solutions for task 1 where she increased her SSB (\%) from $33.3 \%$ to the perfect score. In round one she writes the correct solution that is based on the numbering strategy. As can be seen in line 2, Mary first substitutes the units of the expression $\mathrm{J}=\mathrm{kg} \cdot ? \cdot{ }^{\circ} \mathrm{C}$ into numbers resulting in $8=2 \cdot 2 \cdot 2$. Since the task is to solve for $c$ and $c=2$, she solves for 2 in the numerical expression yielding $2=\frac{8}{2 \cdot 2}$. Then, she re-substitutes units for numbers giving her the correct expression $[c]=\frac{\mathrm{J}}{\mathrm{kg}{ }^{\circ} \mathrm{C}}$ : “Yes, if you take 8, for example, that is 2 times 2 times 2 and $2=8$ divided by 2 times 2 , because that is 8 divided by 4 , and 8 divided by 4 is 2 . So, yes, I think this is the solution". 
Table 6. Mary's solutions for task 1 during algebraic problem-solving in round 1 and 2

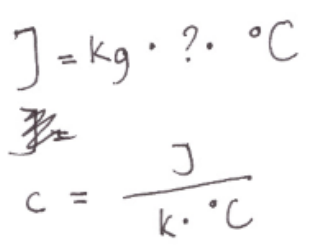

$$
\begin{aligned}
& 8=2 \cdot 2 \cdot 2 \quad \text { Line } \\
& 2=\frac{8}{2 \cdot 2}=\frac{8}{4}=2
\end{aligned}
$$

$$
\begin{aligned}
& Q=m \cdot c \cdot \Delta T \\
& \frac{Q}{m \cdot \Delta T}=\frac{m \cdot c \cdot \Delta T}{m \cdot \Delta T} \quad \text { Line } \\
& c=\frac{Q}{\mathrm{~m} \cdot \Delta T} \\
& {[c]=\frac{\mathrm{J}}{\mathrm{kg} \cdot{ }^{\circ} \mathrm{C}}}
\end{aligned}
$$

Table 7. Sam's basic algebraic skills and symbol sense behavior characteristics per task during algebraic problem-solving in round 1 and 2

\begin{tabular}{ccccccccc}
\hline & $\mathbf{1}$ & $\mathbf{2 a}$ & $\mathbf{2 b}$ & $\mathbf{3}$ & $\mathbf{1}^{\prime}$ & $\mathbf{2 a}^{\prime}$ & $\mathbf{2 b}^{\prime}$ & $\mathbf{3}^{\prime}$ \\
\hline Missed opportunity & 1 & - & - & - & - & - & - & 3 \\
\hline Negative score & - & - & - & 1.5 & - & - & - & - \\
\hline Positive score & 2 & 2 & 2 & 1 & 3 & 2 & 2 & 4 \\
\hline Task max. Score & 3 & 2 & 2 & 4 & 3 & 2 & 2 & 4 \\
\hline SSB (\%) & 66.7 & 100.0 & 100.0 & 62.5 & 100.0 & 100.0 & 100.0 & 100.0 \\
\hline
\end{tabular}

In the second round, she starts with the problem after carefully reading the systematic hints and divides both sides of $Q=m \cdot c \cdot \Delta T$ by $m \cdot \Delta T$ (line $2^{\prime}$ ), and correctly writes the solution:

First, I' $m$ going solve for $c$. Then, I' $m$ going to divide through $m$ and $\Delta T$. This gives $c$ is $Q$ divided by $m$ multiplied by $\Delta T$, and then I'm going to put $c$ in brackets. And then writing all units. I really liked these [systematic] hints.

It can be seen that she applies systematic hints in a well-structured manner. We conclude that although the solution in round one is correct, a rule-based problem-solving approach in which algebraic techniques are used with insight as in the second round is absent.

In general, like Clare, Mary's application of systematic hints transformed her lower scores for the high number of 'Missed opportunity' of round one into positive scores in round two. The []-operator was used correctly in all problems. Because of systematic hints, Mary almost did not use the numbering strategy that was frequently used in the first round. She was quite diligent in reading the systematic hints carefully and applying them in a much more structured manner. Contrary to Clare, she regularly went back to systematic hints of previous problems to use these for other problems. Thus, she seems to be aware of the benefits of the systematic hints. Consequently, the 'activation hint' of task 2a was applied on task $2 \mathrm{~b}$ and 3 . She found the 'activation hints' of task 1 and $2 a$ very useful. This also applies for the 'strategic hint' of task 3 . When triggered just a few more times by systematic hints, Mary may soon make the wholehearted transition from ad hoc strategies to systematic algebraic problem-solving with insight.

\section{Sam}

Sam is the only male student in this study. His grade for mathematics is a 7.9 and for for physics a 5.9. In round one, he faced some difficulties with questions 1 and 3, and only used a hint for task 3 . Sam did not use hints in round two. Furthermore, for both rounds he did not use ad hoc approaches.

His symbol sense behavior characteristics for both rounds are illustrated in Table 7. Concerning both rounds, Sam has only one 'Missed opportunity' in the first where he overlooked an local salience procedure. Consequently, the percentage of his total number of 'Missed opportunity' is low, i.e. $9.1 \%$ of the perfect score and even $0 \%$ in round two.

These percentages are in contrast to those of the other students. Indeed, they used ad hoc strategies and overlooked procedures for many times. Sam did did not use ad doc strategies. Together with one overlooked procedure above, this explains the discrepancy between them. In addition to his lowest SSB (\%) of $62.5 \%$ in round one for task 3 , his score was maximal for tasks $2 \mathrm{a}$ and $2 \mathrm{~b}$. These numbers are in contrast with Clare and Mary who both had zero scores in round one. In round two, Sam even maximized task 1 and 3 by gaining the perfect score. Like Mary, his OBAS (\%) was already $100.0 \%$ in the first round and did not change in the second. Since his symbol sense behavior characteristics were much better than the other students, he had also the highest OSSB (\%) that improved from $77.3 \%$ in the first to the perfect score in the second round. So Sam is the only student who's basic 
Table 8. Sam's solutions for task 3 during algebraic problem-solving in round 1 and 2

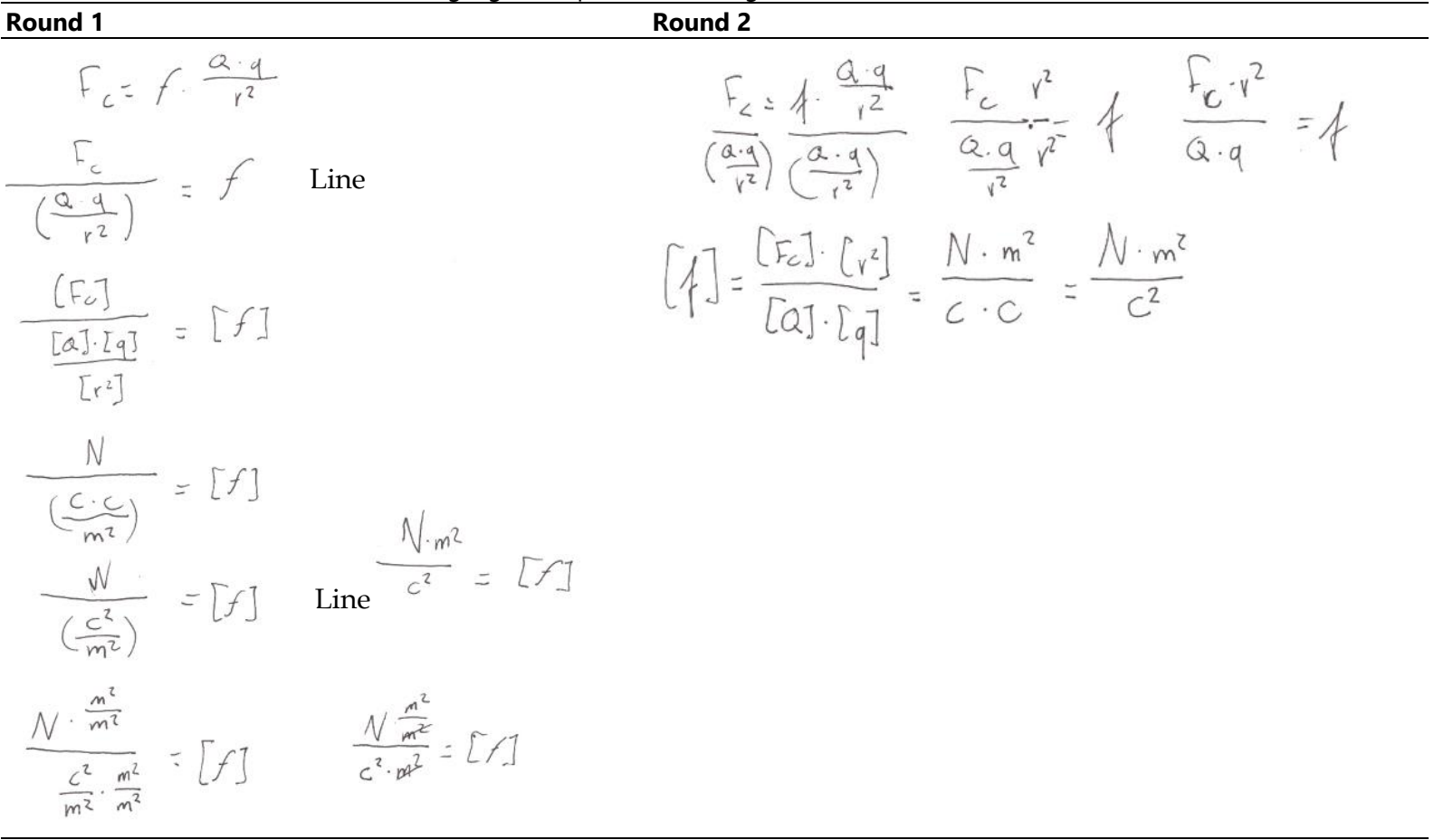

algebraic skills and symbol sense behavior were already sufficient in the first round. He is also the only student with a perfect score for all tasks in the second round, implying the he increased his scores maximally.

In Table 8, we displayed Sam's work for task 3 where he improved his SSB (\%) from $62.5 \%$ to a flawless score. In the first round he correctly divides both sides of $F_{C}=f \cdot \frac{Q \cdot q}{r^{2}}$ through $\frac{Q \cdot q}{r^{2}}$ yielding a term with double divisions $f=\frac{F_{C}}{\frac{Q \cdot q}{r^{2}}}$ in line 3. Therafter, he intended to stop working on this task, but then proceeded when the interviewer asks him how to get rid of the double divisions (hint). After substituting the quantities in this formula, he tries to simplify this expression. Still, he is not able to get rid of those divisions. Sam suspects that he might cross out $m^{2}$ in line 5 . On the left side in line 6 he multiplies both the numerator and the denominator of this expression with $\frac{\mathrm{m}^{2}}{\mathrm{~m}^{2}}$. On the the right side of the same line, Sam divides away $m^{2}$, but mentions that he is not sure whether this procedure is correct: "I don't know whether this is allowed. If I did it right. May be I can cross-out some terms. I don't know whether it is allowed". In fact, crossing-outs these terms is a guess, without any mathematical understanding of this procedure. In the next round, he correctly divides away both sides of the initial formula through $\frac{Q \cdot q}{r^{2}}$. Subsequently, he multiplies the new expression by $\frac{r^{2}}{r^{2}}$ and finds $f=\frac{F_{C} \cdot r^{2}}{Q \cdot q}$. After applying the []-operator and substituting units in the formula he finds the correct solution. Interestingly, Sam applies the 'activation hint' of problem 2a: “I didn't really use this [systematic] hint. I used that of a previous one [problem 2a]. Although I understand the [systematic] hint, I didn't use it for this task".

Overall, except for the divisions above, Sam did not encounter serious transfer problems. Contrary to Clare and Mary, he did not use ad hoc strategies a single time. Remarkably, he already used both the []-operator and systematic algebraic strategies in the first round. He found the 'activation hint' of task 2 a very useful and made repeated statements that the algebraic skills underlying the 'activation hints' were already taught in mathematics class not too long ago. Indeed, in the second round he used those strategies effectively and maximized his performance with a perfect OSSB (\%). Sam is on the right track; he justifies and develops his already existing tendency to use ad hoc approaches.

\section{OSSB (\%) in Both Rounds}

To gain more insight in the effectiveness of our intervention, in Figure 2 we displayed OSSB (\%) of the first round next to that of the second. To distinguish between both rounds, like in Table 3, 5 and 7 where we used apostrophes for the tasks in the second round, here we used OSSB (\%)'. This is illustrated in Figure 2. Since OBAS $(\%)$ and OBAS (\%)' were already incorporated in OSSB (\%) and OSSB (\%)' respectively in the previous subsection, we did not depict them. 


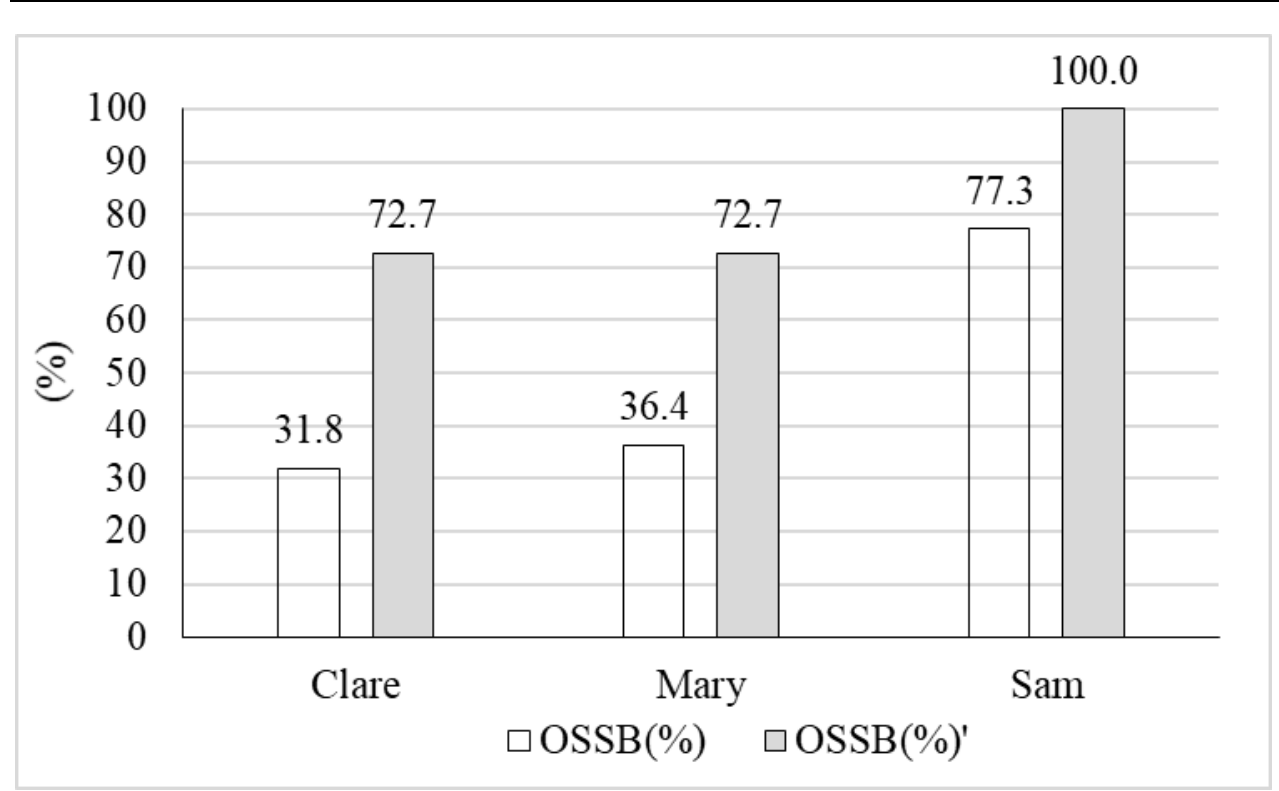

Figure 2. OSSB (\%) of the first round next to OSSB (\%)' of the second

For all students there is a major increase in OSSB (\%) from the first to the second round. While Sam is the only student having both sufficient procedural fluency (OBAS $(\%) \geq 75 \%$ ) and symbol sense behavior (OSSB $(\%) \geq 72.7$ $\%)$ already in round one, the other students demonstrated that in the second. We also calculated the ratio $\frac{\text { OSSB (\%) }}{\text { OSSB (\%) }}$ that gives the relative increase of students' OSSB (\%) over both rounds and therefore may be seen as the effectiveness of our intervention by means of shift-problems. For Clare we have $\frac{\text { OSSB (\%) })}{\text { OSSB (\%) }}=\frac{72.7}{31.8}=2.3$, for Mary $\frac{72.7}{36.4}=2.0$ and for Sam $\frac{100.0}{77.3}=1.3$. These numbers confirm our earlier findings: the systematic hints provided in the second round led to a major increase in students' symbol sense behavior.

We also calculated $\langle$ OSSB (\%) $\rangle$ and $\langle\text { OSSB }(\%)\rangle^{\prime}$ where the symbols \langle\rangle refer to the average. Therefore, $\langle$ OSSB $(\%)\rangle$ is the average of OSSB (\%) among all students, and $\langle\text { OSSB (\%) }\rangle^{\prime}$ the average OSSB (\%)' among all students. This number increases from $48.5 \%$ in the first to $81.8 \%$ in the second round. So, while the average OSSB (\%) of all students was insufficient in round one, this changed to sufficient in the second, again indicating the effectiveness of how we implemented shift-problems.

\section{Average SSB (\%) per Task in Both Rounds}

In Figure 3 we displayed $\langle$ SSB (\%) $\rangle$, i.e. the average SSB (\%) per task among all students in round one, and analogously $\langle\text { SSB }(\%)\rangle^{\prime}$ for round two. Round one may be characterized by two regimes: the very low scores for task $1(44 \%)$, task $2 \mathrm{~b}(33.3 \%)$ and task $3(37.5 \%)$, and the very high score for task $2 \mathrm{a}(91.7 \%)$. Furthermore, because of the criterion OSSB $(\%) \geq 72.7 \%$, $\langle$ SSB $(\%)\rangle \geq 72.7 \%$ was considered sufficient. Only task 2 a met this criterion. Indeed, contrary to task $2 \mathrm{a}$, the other tasks contained a combination of many ad hoc approaches and overlooked procedures, strongly impeding students' OSSB (\%). The next round may be characterized by the high regime including task $1(77.8 \%)$, task $2 \mathrm{~b}(83.3 \%)$ and task $3(75.0 \%)$, and the highest regime including task $2 \mathrm{a}(100.0 \%)$. In the next round, all these tasks met the criterion above, with task $2 a$ even reaching the perfect score. This finding confirms what we have seen before: in round two students adopted systematic hints and applied them quite succesfully.

On the individual task level, for task 1 in the first round students mainly missed points because they did not divide both sides of $Q=m \cdot c \cdot \Delta T$ through $m$ and $\Delta T$. In addition, Mary used the numbering strategy a few times. The sufficient $\langle$ SSB $(\%)\rangle$ in the next round was mainly due Mary and Sam who flawlessly applied the systematic hints.

As to task 2a, students' solid domain of basic algebraic skills resulted in excellent performances for both basic algebraic skills procedures. The already sufficient $\langle$ SSB $(\%)\rangle$ in round one reached the perfect score in the second. The perfect scores in both rounds make it very difficult to argue to which extent the systematic hints were useful.

Task $2 \mathrm{~b}$ consisted of two pattern salience procedures. Prior to the interviews, we expected this task to be the most difficult problem. Indeed, this was the case for round one where except for Sam the other students struggled seriously. Starting with $R_{\text {therm }}=\frac{d}{\lambda \cdot A}$, they did not see the inverse relationship $\frac{1}{R_{\text {therm }}}=\frac{\lambda \cdot A}{d}$. They also did not see that 


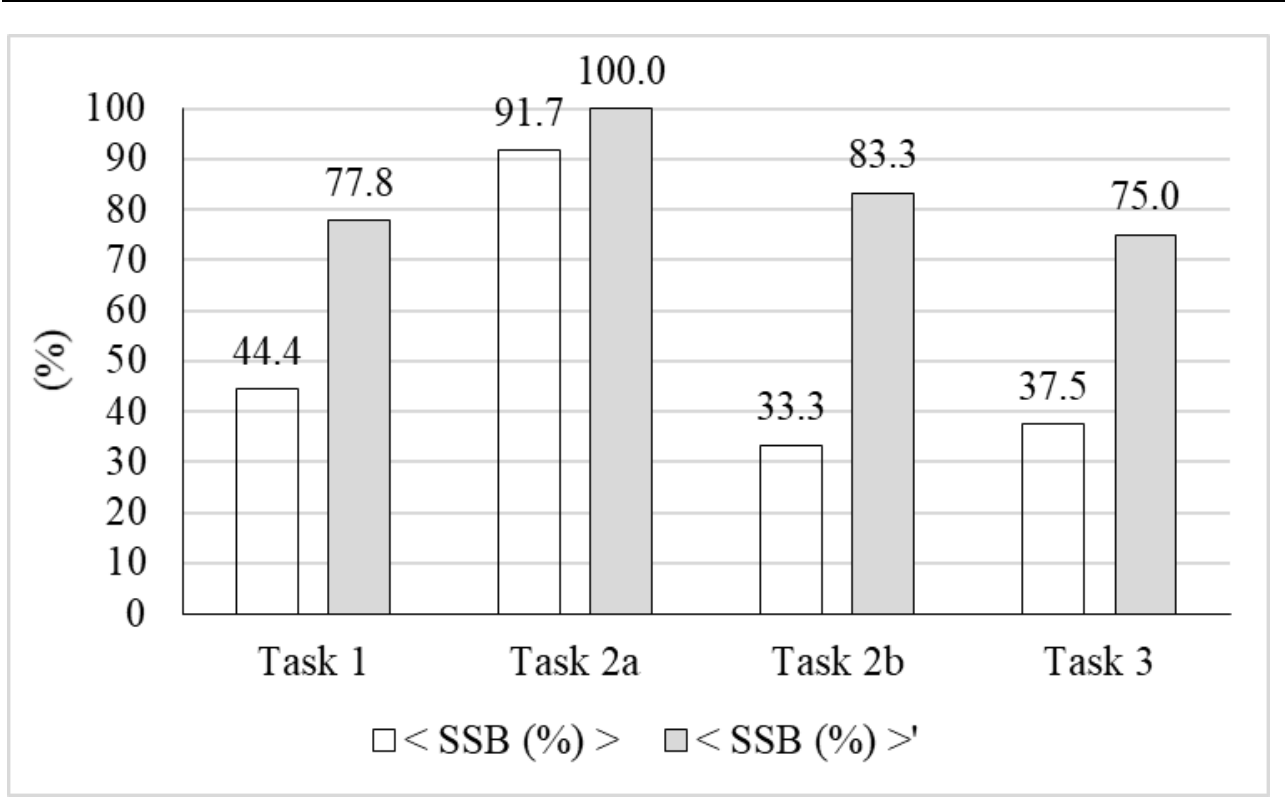

Figure 3. $\langle\mathrm{SSB}(\%)\rangle$ per task among all students next to $\langle\mathrm{SSB}(\%)\rangle^{\prime}$ of the second

$P=\frac{\lambda \cdot A \cdot \Delta T}{d}$ in the second procedure can be written as $P=\frac{\lambda \cdot A}{d} \cdot \Delta T$. So, although the first procedure would be correct, they would perform incorrectly on the second. Thereafter, 〈SSB (\%)〉 improved, where Mary and Sam used systematic hints that were mainly adopted from other questions such as 2a. Unfortunately, Clare did not understand the purpose of them. In short, we may say that the systematic hints provided for task $2 \mathrm{~b}$ were not helpful for students. It might be the case that these systematic hints should be ajusted into more appropriate ones.

Task 3 is the question containing the largest number of variables. Hence, compared to other questions, it is also the task requiring the largest number of procedures. We observed that students struggled with dividing away the fraction $\frac{Q \cdot q}{r^{2}}$ in $F_{C}=f \cdot \frac{Q \cdot q}{r^{2}}$. Their work involving ad hoc strategies and overlooked procedures, strongly impeded $\langle$ SSB (\%) $\rangle$ for this task. It may be the case that especially Mary and Sam lost control, got stuck because of the large number of variables and failed in making the final step; Mary, since she substituted numbers for $F_{C}=f \cdot \frac{Q \cdot q}{r^{2}}$ to understand the arrangement of the valid outcome for $f$, and Sam since he could not get rid of the doubled division term. Clare overlooked procedures because she immediately swapped the fraction $\frac{Q \cdot q}{r^{2}}$ into the denominator without understanding the mathematical legitimacy of this step, i.e. $f=\frac{F_{C}}{\left(\frac{Q \cdot q}{r^{2}}\right)}$. During the second round, all students used systematic hints to solve this task. Remarkably, instead of the 'activation hint' of this question, students used that of task $2 \mathrm{a}$ and multiplied both sides of $F_{C}=f \cdot \frac{Q \cdot q}{r^{2}}$ with $\frac{r^{2}}{r^{2}}$. Mary, however, lost points since she also used the numbering strategy.

\section{CONCLUSION AND DISCUSSION}

The aim of this study was to examine the possibility to improve students' symbol sense behavior through activation of prior mathematical knowledge during algebraic problem-solving in physics. To this extent we developed shift-problems. The main difference with earlier studies investigating shift-problems (Palha, Dekker, \& Gravemeijer, 2015; Palha, Dekker, Gravemeijer, \& van Hout-Wolters, 2013) is that they were for the first time designed outside mathematics education, i.e. in physics education. In addition, except for the study examining symbol sense behavior in physics problems (Turşucu et al., 2018b), this is the second time that this concept was studied outside mathematics education. Also, our tasks consisted of expressions with variables representing real, measurable quantities in physics. Other studies used abstract variables in mathematics without meaning in real life.

Regarding operationalization of symbol sense behavior, we followed the method that we have developed to research students' basic algebraic skills and sensitivity towards gestalt view and visual salience by means of local salience and pattern salience of algebraic expressions (Turşucu et al., 2018b). To assess students' work, we used traditional pen-and-paper settings present in previous studies (Arcavi 1994, 2005; Wenger 1987) and not a digital environment such as Bokhove and Drijvers (2010). Unlike these studies, which mainly have a qualitative character, we assessed students' work both qualitatively and quantitatively, i.e. we determined students' performance on procedures concerned with basic algebraic skills and symbol sense behavior were both quantized. To this extent, 
we used the coding scheme of Table 2 together with the systematic solution set including clearly worked out procedures in the Appendix. Furthermore, we analysed videotaped data in detail following the seven consecutive steps in the theoretical model of Powell et al. (2003). Except for the study of (Turşucu et al., 2018b), such detailed analysis is not present in earlier studies.

As to the applicibility of our systematic algebraic problem-solving approach with insight involving activation of mathematical knowledge, we expect this approach to be applicable to other other science subjects. (and even other disciplines). For instance, solving for a variable in both Poiseuille's law $Q=\frac{\pi \cdot P \cdot r^{4}}{8 \cdot \gamma \cdot l}$ in biology and the equilibrium equation $K_{e q}=\frac{[C]^{c} \cdot[D]^{d}}{[A]^{a} \cdot[D]^{d}}$ in chemistry.

Similar to Bokhove and Drijvers (2010), the tasks were carefully selected to trigger students' problem-solving and to offer deeper understanding of students' performance including basic algebraic skills and symbol sense behavior. Indeed, this was the case in both rounds contributing to the internal validity of this study.

Concerning the sub question (A) "To what extent do students in upper secondary education demonstrate symbol sense behavior when solving algebraic physics problems that occur in their physics textbooks?", two of the three students' symbol sense behavior was insufficient in the first round. This was mainly due to overlooked procedures and the application of ad hoc strategies lacking a rule-based problem-solving approach in which algebraic techniques are used with insight. Regarding sub question (B) "To what extent do students in senior pre-university education demonstrate symbol sense behavior when solving the same algebraic physics problems that occur in their physics textbooks after activation of prior mathematical knowledge?", students demonstrated sufficient symbol sense behavior. This implies that students adopted the systematic hints that we provided appropriately and increased their symbol sense behavior in the second round. They strongly benefited from our intervention including activation of mathematical knowledge where we offered rule-based systematic hints with insight, especially algebraic techniques that were treated in a similar way to how they were learned in their mathematics textbooks (Reichard et al., 2014). Therefore, our central research question "How can activation of prior mathematical knowledge be used effectively to improve students" symbol sense behavior in senior pre-university education when solving algebraic physics problems?" can be answered that using algebraic skills in the same way as in mathematics textbooks to activate prior mathematical knowledge was quite effective for our sample of three students. Indeed, they increased their OSSB (\%) from $48.5 \%$ in the first to 81.8 $\%$ in the second round. To generalize this result to the whole Dutch context, one would need replication using a larger sample.

We have seen that using ad hoc strategies may help students to solve basic algebraic problems, but there are risks for the longer term, especially in more sophisticated problems requiring insight. Applying them depends on the approval of an authority such as a teacher or a textbook explaining them what is mathematically correct and what is not. As a consequence, mathematics can become a set of unconnected strategies lacking conceptual understanding. Since students generally do not know the boundaries of ad hoc approaches, they also do not know where they apply and where not. Therefore, ad hoc strategies are harmful for the application of algebraic skills with insight, corroborating and extending earlier studies (e.g. Drijvers et al., 2011; Roorda, 2012; Turşucu et al., 2018b). Instead of such strategies, students should use systematic algebraic strategies involving a rule-based problem-solving approach in which algebraic skills, especially algebraic techniques are used with insight as in the systematic solution set in Appendix. If our findings above hold up through replication for sufficiently large samples, then we recommend that these findings they should be considered and implemented by the mathematics and science audience, especially curriculum developers, mathematics and physics teachers, mathematics and science teacher educators and textbook publishers aiming to foster transfer between both subjects and strengthen students' experiencing more coherence across these subjects.

As to the first group, we suggest using content standards that emphasize the importance of using algebraic skills with insight as described in the mathematics curriculum (Netherlands institute for curriculum development, 2018). Prior mathematical knowledge should be activated through the same pedagogy of applying algebraic skills, especially algebraic techniques that occur in mathematics curricula. Content standards of the latter should pay attention to the importance of algebraic physics problems analogous to mathematics problems.

Moreover, independent of whether those curricula are centralized and determine the textbook content, we recommend that it is probably better that mathematics and physics textbook publishers avoid ad hoc strategies such as the numbering strategy. Instead, mathematics textbooks should pay attention to systematic algebraic strategies where algebraic skills, especially different forms of algebraic techniques are emphasized, for example 'inverting both sides of the equation. In addition, we suggest adopting science context such as physics problems. In terms of CMSE, it is probably better that physics problems in physics textbooks are introduced through paragraphs containing analogous mathematics problems that students have learned in mathematics class. Systematic hints can be taught together with systematic procedures as in the solution set. Even though CMSE is important, it requires sufficient organization of the learning process in order to achieve a logical learning line across 
both subjects. In practice, unfortunately, it still happens that certain mathematical concepts are used in physics class before they were introduced in mathematics class (Alink et al., 2012; Roorda, 2012; Turşucu et al., 2017, 2018a).

On the individual level, physics teachers explaining relevant basic mathematics should be a pre-requisite for pre-service teachers involved in science teacher education programmes leading to a teaching qualification - this does not apply for mathematics teachers, because basic mathematical knowledge is considered to be part of mathematics teacher education programmes. Besides the application of systematic algebraic strategies, we recommend mathematics teachers to refer to the importance of mathematics in physics. For instance, mentioning that mathematics is applied in science subjects such as physics, writing mathematics expressions next to physics formulas may improve transfer and strengthen students experiencing more coherence across these subjects (Turşucu et al., 2018a). Physics teachers can, for example emphasize that algebraic skills learned in mathematics class are applied in physics class and also use the same pedagogy of systematic algebraic approaches as mathematics teachers. Such issues require teachers from both departments to communicate with each other. However, internationally teachers' curricula are overloaded (e.g. Lyons, 2006). Thus, such collaborative efforts between teachers should be feasible to adopt in teaching practice. In this respect, we suggest individual efforts on a small scale during informal meetings.

Regarding mathematics and science teacher educators, we recommend that both of them make 'in service' and 'preservice' teachers aware of the harmful ad hoc strategies and their underlying mechanisms. Moreover, there is the possibility to develop partly integrated teaching materials emphasizing the danger of such strategies for students and propose common identical pedagogical approaches to combat ad hoc strategies through systematic algebraic approaches. Furthermore, the application of algebraic skills with insight that becomes visible during basic algebraic skills and symbol sense behavior procedures is discussed in mathematics education. We suggest science teacher educators to explain this concept for problem-solving in physics in relation to the importance of activation of prior mathematical knowlegde to teachers and future teachers.

The concepts of basic algebraic skills and symbol sense behavior are intertwined (see Figure 1). Hence, it is not easy to recognize on which concept students rely (Bokhove \& Drijvers, 2010). Nevertheless, we succeeded in recognizing them in both rounds quite easily using the numerical criteria OSSB $(\%) \geq 72.7 \%$ and SSB $(\%) \geq 72.7 \%$. In the same way with the OBAS $(\%) \geq 75 \%$ criterion we were able to observe and measure basic algebraic skills procedures. Moreover, investigation of OBAS (\%) and OSSB (\%) separately might indicate that we decoupled both concepts. In fact, we studied OBAS (\%) to gain deeper understanding into the degree to which students demonstrated a solid domain of basic algebraic skills. On the other hand, OBAS (\%) was already part of OSSB (\%), keeping their intertwinement intact.

As for students who encountered problems applying mathematics in physics, the findings above, especially in the first round corroborate and extend earlier studies (e.g. Dierdorp et al., 2014; Rebello et al., 2007; Roorda et al., 2015; Turşucu et al., 2018a; Wong, 2018), contributing to the relevance of this study. This also applies for previous studies on learning and instruction emphasizing the importance of activation of pre-knowledge for better students' achievements (e.g. Hailikari, Katajavuori, \& Lindblom-Ylanne, 2008; Roorda, 2012). Indeed, our intervention with shift-problems activating pre-mathematical knowledge improved the students' performance in our sample. Again, we emphasize that replication of our results in a larger sample is needed to generalize this result from sample to population.

\section{Limitations and Further Recommendations}

For this the purpose of this qualitative study, we aimed at interviewing three students. Indeed, over two rounds of problem-solving, we expected that this would provide us sufficient data. This was indeed the case. Therefore, we ignored a gender ratio of $50 \%-50 \%$. Our extended literature research reveals that there are no indications that a small sample with an uneven gender ratio would yield different results. It is very likely that students' OBAS (\%) and OSSB (\%) in both rounds are related to a similar knowledge domain at the start of these interviews and their mathematics and physics grades. Regardless of gender, such samples may have generated comparable results. Therefore, we do not view the composition of our sample as a limiting factor, and we note that this finding contributes to the internal validity of this study. Our sample is too small to justify generalizations of our results to the population.

The selection criteria in this study were so strong that we only found one school meeting these requirements. Still, we found exactly three students, i.e. the number of students we aimed for. Among them, only one student whose physics grade was 5.9 (instead of <5.5) did not perfectly meet these criteria. We selected this student because his (Sam) physics grade was in the 'danger zone'. Contrary to the other female students whose OSSB (\%) scores were insufficient in the first round, Sam's OSSB (\%) performance was sufficient in both rounds. This may lead to the following question: "Would these scores have been insufficient if his physics grade was insufficient?". This may or may not be the case, but this question is too difficult to answer. For instance, he may have repeated his class and 
improved his problem-solving skills. On the other hand, his mathematics grade of 7.9 that is approximately 1.0 higher than the other students, may also not explain Sam's performance. Indeed, in the study of Turşucu et al. (2018b) that only assessed symbol sense behavior in algebraic physics problems, students with similar mathematics grades performed quite poorly on the transfer tasks leading to both insufficient OBAS (\%) and OSSB (\%) scores. Furthermore, the selection of students with a combination of sufficient knowledge of mathematics and poor knowledge of physics might be both a strength and a weakness in this study. Indeed, because of such combination, activation of prior mathematical knowledge may be relatively assured. What would happen, for example, when our sample consisted of students whose mathematics and physics performances are nearly equal? This is a very relevant question, since this extends the scope of this study providing insight in the students' algebraic problemsolving abilities with sample characteristics not examined in this study. These samples contain students who have both sufficient mathematics and physics grades, and students with both insufficient mathematics and physics grades. How would such students perform comparing the first and the second round? And, what can be learned when all the three groups (including this current study) are viewed together?

Except for additional information about the introductory task and the systematic hints provided in the second round, students were asked to solve the same questions in both rounds. Could this have caused undue bias? We think that this might indeed be the case. Hence, it would be interesting to use the same algebraic problems in two different groups, each containing large number of students. Following this study, the time between the first and the second round is kept at two weeks. Since the intervention is only applied in one group, the results in both groups can be compared to each other. This can provide insight in the extent to which replication of the same problems in both rounds may have caused undue bias. In addition to applying algebraic skills from mathematics to solve algebraic physics problems, it can be worthwhile to investigate the usefulness of systematic hints in other areas of physics, for example the application of trigonometry in phyiscs problems. We also think that applying systematic hints can be valuable for other science subjects, for example solving aforementioned algebraic problems such as Poiseuille's law in biology and the equilibrium equation in chemistry.

With regard to representativity of our sample, these students are not representative for the Dutch context. For instance, their problem-solving skills and symbol sense behavior characteristics are too different. In addition, the findings following from this study cannot be extended for the Dutch population, neither to other countries outside the Netherlands. Indeed, our sample size is simply too small. Yet, concerning pedagogical approaches to algebraic problem-solving in grade-10 mathematics and physics textbooks, there may be similarities between the Netherlands and other countries with national final examinations which are described in curricula through the general educational core goals and the more specific standards, shaping textbook driven mathematics and physics teachers who teach those textbooks to their students.

In this study, the students were provided systematic hints in the second round to improve their symbol sense behavior. Other hints were offered when students got stuck to help them proceed. These hints were neutral, meaning that they were as unobstructive as possible. For instance, during task 3 of the first round (see line 5 of Table 8), Sam got stuck and mentioned "I do not know whether I can further simplify this term, but at least until I can". The interviewer responded: "Can you simplify it one step further? To avoid having two divisions in that term?". Thereafter, even he performed incorrectly, Sam continued his work. Other hints in this study were also neutral. Some of the tasks were not read carefully by students, especially the systematic hints. In addition, for most of the tasks the students worked too fast resulting in sloppy mistakes that may have been overcome if they had carefully re-examined their work. Hence, we suggest to adjust the interview protocol and incorporate two design principles: it should explicate that students should read the questions very carefully and re-examine their work after they finished their tasks. Earlier studies state that such adjustments may add to students' meta-cognitive skills (Hattie \& Timperley, 2007; Shute, 2008). This in turn can improve students' transfer.

The findings above offer insight in the relation between procedural fluency and symbol sense, and how these concepts should be treated in effective teaching practice. Our findings show the effectiveness of using sytematic algebraic strategies involving a rule-based approach where basic skills are used with insight to combat problems that require unusual reasoning. The discussion is not about focussing on basic skills or insight. Instead, both basic skills and symbol sense should be taught in an integrated manner. This confirms the statement "Without insight, there is no skill, and without skill, there is no insight" (Drijvers, 2011, p. 141).

Two of the three students used harmful ad hoc strategies. One of them mentioned that she had learned the swapping approach from her mathematics teacher, which might indicate that her teacher taught this strategy from her mathematics textbook (Reichard et al., 2014a). This method has the largest market share in the Netherlands, influencing a large number of highly textbook-driven teachers teaching them to their students (Stein \& Smith, 2010; van Zanten \& van den Heuvel-Panhuizen, 2014). Therefore, we suggest further research investigating to which extent such strategies are involved in this type of textbook series. We strongly recommend researchers sharing their findings with the textbook publisher. If needed, we suggest making publishers aware by pointing out the risks for teachers and students such strategies entail. This may also be a point of attention for grade 9. Grade-10 students' 
lack of insight in the application of algebraic skills in round one may be due to insufficient emphasis on a rulebased problem-solving approach on conceptual understanding in grade 9 . Therefore, as for textbooks we recommend examining grade 9 textbooks to elaborate on this matter.

\section{REFERENCES}

Accounts.magister.net. (2019, November 22). Retrieved from https:/ / accounts.magister.net/

Alink, N., Asselt, R. van, \& Braber, N. den (2012). Samenhang en afstemming wiskunde en de profielvakken [Coherence between mathematics and the profile subjects]. Utrecht / Enschede, The Netherlands: cTWO / SLO.

Andrá, C., Lindström, P., Arzarello, F., Holmqvist, K., Robutti, O., \& Sabena, C. (2015). Reading mathematics representations: An eye-tracking study. International Journal of Science and Mathematics Education, 13(2), 237259. https:// doi.org/10.1007/s10763-013-9484-y

Arcavi, A. (1994). Symbol Sense: Informal Sense-Making in Formal Mathematics. For the Learning of Mathematics, 14(3), 24-35.

Arcavi, A. (2005). Developing and using symbol sense in mathematics. For the learning of mathematics, 25(2), 42-47.

Berlin, D. F., \& White, A. L. (2010). Preservice mathematics and science teachers in an integrated teacher preparation program for grades 7-12: A 3-year study of attitudes and perceptions related to integration. International Journal of Science and Mathematics Education, 8(1), 97-115. https:/ / doi.org/10.1007/s10763-009-9164-0

Berlin, D. F., \& White, A. L. (2012). A longitudinal look at attitudes and perceptions related to the integration of mathematics, science, and technology education. School science and mathematics, 112(1), 20-30. https:// doi.org/10.1111/j.1949-8594.2011.00111.x

Berlin, D. F., \& White, A. L. (Eds.). (2014). Initiatives in Mathematics and Science Education with Global Implications. Columbus, OH: International Consortium for Research in Science and Mathematics Education.

Bokhove, C., \& Drijvers, P. (2010). Symbol sense behavior in digital activities. For the Learning of Mathematics, 30(3), 43-49.

Bryman, A. (2015). Social research methods (5th ed.). Oxford, UK: Oxford University Press.

Charters, E. (2003). The use of think-aloud methods in qualitative research an introduction to think-aloud methods. Brock Education Journal, 12(2). https:/ / doi.org/10.26522/brocked.v12i2.38

Dierdorp, A., Bakker, A., van Maanen, \& J., Eijkelhof, H. (2014). Meaningful statistics in professional practices as a bridge between mathematics and science: an evaluation of a design research project. International Journal of STEM Education, 1(9), 1-15. https:/ / doi.org/10.1186/s40594-014-0009-1

Drijvers, P. (Ed.) (2011). Secondary algebra education. Revisiting topics and themes and exploring the unknown. Rotterdam, The Netherlands: Sense.

Furner, J., \& Kumar, D. (2007). The Mathematics and Science Integration Argument: A stand for Teacher Education. Eurasia Journal of Mathematics, Science $\mathcal{E}$ Technology Education, 3(3), 185-189. https:/ / doi.org/10.12973/ ejmste/75397

Geiger, V. S, Muir, T., \& Lamb, J. (2016). Video-stimulated recall as a catalyst for teacher professional learning. Journal of Mathematics Teacher Education, 19(5), 457-475. https:/ / doi.org/10.1007/s10857-015-9306-y

Girvan, C., Conneely, C., \&Tangney, B. (2016). Extending experiential learning in teacher professional development. Teaching and Teacher Education, 58(206), 129-139. https:/ / doi.org/10.1016/j.tate.2016.04.009

Goldin, G. A. (2000). A scientific perspective on structured task-based interviews in mathematics education research. In A. Kelly, \& R. Lesh (Eds.), Handbook of research design in mathematics and science education (pp. 517-545). Mahwah, NJ: Lawrence Erlbaum.

Hailikari, T., Katajavuori, N., \& Lindblom-Ylanne, S. (2008). The relevance of prior knowledge in learning and instructional design. American Journal of Pharmaceutical Education, 72(5), 113. https://doi.org/10.5688/aj7205113

Haskell, R. E. (2001). Transfer of learning: Cognition, instruction and reasoning. San Diego, CA: Academic Press.

Hattie, J., \& Timperley, H. (2007). The power of feedback. Review of Educational Research, 77(1), 81-112. https:// doi.org/10.3102/003465430298487

Honey, M., Pearson, G., \& Schweingruber, H. (Eds.). (2014). STEM integration in K-12 education: Status, prospects, and an agenda for research. Washington, DC: National Academies Press.

Jonassen, D. H. (Ed.). (2017). Learning to solve complex scientific problems. Routledge. https://doi.org/10.4324/9781315091938 
Kilpatrick, J., Swafford, J., \& Findell, B. (2001). Adding It Up: Helping Children Learn Mathematics. Washington, DC: National Academy Press.

Leahy, S., \& Wiliam, D. (2012). From teachers to schools: scaling up professional development for formative assessment. Assessment and learning, 2, 49-71. https:/ / doi.org/10.4135/9781446250808.n4

Lobato, J. (2003). How design experiments can inform a rethinking of transfer and vice versa. Educational Researcher, 32(1), 17-20. https:// doi.org/10.3102/0013189X032001017

Lobato, J., Rhodehamel, B., \& Hohensee, C. (2012). “Noticing” as an alternative transfer of learning process. Journal of the Learning Sciences, 21(3), 433-482. https:/ / doi.org/10.1080/10508406.2012.682189

Lyons, T. (2006). Different countries, same science classes: Students' experiences of school science in their own words. International Journal of Science Education, 28(6), 591-613. https:/ / doi.org/10.1080/09500690500339621

Maher, C. A., \& Sigley, R. (2014). Task-based interviews in mathematics education. In S. Lernman (ed.), Encyclopedia of Mathematics Education. Dordrecht, The Netherlands: Springer Science+Business Media. https:/ / doi.org/10.1007/978-94-007-4978-8

Mestre, J. P. (Ed.). (2005). Transfer of learning from a modern multidisciplinary perspective. Greenwich, CT: Information Age.

Ministry of Education, Culture and Science. (2019, November 23). Retrieved from https:/ / www.government.nl/ministries/ministry-of-education-culture-and-science

National Research Council. (2013). The mathematical sciences in 2025. Washington, DC: National Academies Press.

Netherlands institute for curriculum development. (2018, May 27). Retrieved from http:/ /international.slo.nl

OECD. (2018, September 3). Retrieved from http:/ / www.oecd.org/

Ottink, et al. (2014). Systematische Natuurkunde, $8^{e}$ editie basisboek, 4 vwo [Systematic Physics, $8^{\text {th }}$ edition basic textbook, first year senior pre-university education]. Amersfoort, The Netherlands: ThiemeMeulenhoff.

Palha, S., Dekker, R., \& Gravemeijer, K. (2015). The effect of shift-problem lessons in the mathematics classsroom. International Journal of Science and Mathematics Education, 13(6), 1589-1623. https:/ / doi.org/10.1007/s10763014-9543-Z

Palha, S., Dekker, R., Gravemeijer, G., \& Van Hout-Wolters, B., (2013). Developing shift problems to foster geometrical proof and understanding. Journal of Mathematical Behaviour, 32(2), 142-159. https://doi.org/10.1016/j.jmathb.2012.12.002

Powell, A. B., Francisco, J. M., \& Maher, C. A. (2003). An analytical model for studying the development of learners' mathematical ideas and reasoning using videotape data. The journal of mathematical behavior, 22(4), 405-435. https:/ / doi.org/10.1016/j.jmathb.2003.09.002

Rebello, N. S., Cui, L., Bennett, A. G., Zollman, D. A., \& Ozimek, D. J. (2007). Transfer of learning in problem solving in the context of mathematics and physics. Learning to solve complex scientific problems, 223-246. https:/ / doi.org/10.4324/9781315091938-10

Redish, E. F. (2017). Analysing the competency of mathematical modelling in physics. In Key Competences in Physics Teaching and Learning (pp. 25-40). Springer, Cham. https://doi.org/10.1007/978-3-319-44887-9_3

Reichard, L. A., Dijkhuis, J. H., Admiraal, C. J., Vaarwerk te, G. J., Verbeek, J. A., Jong de, G., ... Voets, S. (2014). Getal en Ruimte, wiskunde A, deel 1 [Numbers \& Space, mathematics A, part 1]. Houten, The Netherlands: EPN.

Rittle-Johnson, B., Schneider, M., \& Star, J. R. (2015). Not a one-way street: Bidirectional relations between procedural and conceptual knowledge of mathematics. Educational Psychology Review, 27(4), 587-597. https:/ / doi.org/10.1007/s10648-015-9302-x

Roorda, G. (2012). Ontwikkeling in verandering; ontwikkeling van wiskundige bekwaamheid van leerlingen met betrekking tot het concept afgeleide [Development of 'change'; the development of students' mathematical proficiency with respect to the concept of derivative]. (Doctoral dissertation). Retrieved from https:/ / www.rug.nl/staff/g.roorda/ proefschriftgerritroorda.pdf

Roorda, G., Goedhart, M., \& Vos, P. (2014). An actor-oriented transfer perspective on high school students' development of the use of procedures to solve problems on "rate of change". International Journal of Science and Mathematics Education, 13(4), 863-889. https:/ / doi.org/10.1007/s10763-013-9501-1

Schmidt, W. H., Wang, H. C., \& McKnight, C. C. (2005). Curriculum coherence: An examination of U.S. mathematics and science content standards from an international perspective. Journal of Curriculum Studies, 37(5), 525559. https:// doi.org/10.1080/0022027042000294682

Schoenfeld, A. H. (2016). 100 Years of curriculum history, Theory, and Research. Educational Researcher, 45(2), $105-$ 111. https:/ / doi.org/10.3102/0013189X16639025 
Schoenfeld, A. H., \& Sloane, A. H. (Eds.). (2016). Mathematical thinking and problem solving. London, United Kingdom: Routledge. https:// doi.org/10.4324/9781315044613

Shute, V. J. (2008). Focus on formative feedback. Review of educational research, 78(1), 153-189. https:/ / doi.org/10.3102/0034654307313795

Sonneveld, et al. (2014). Overal Natuurkunde 4 vwo, $4^{e}$ druk [Everywhere Physics, $4^{\text {th }}$ edition, first year of upper secondary education]. Groningen, The Netherlands: Noorhoff Uitgevers B.V.

Stein, M. K., \& Smith, M. S. (2010). The influence of curriculum on student learning. In B. Reyes, R. Reyes, \& R. Rubenstein (Eds.), Mathematics curriculum: Issues, trends and future directions (pp. 351-362). Reston, VA: NCTM.

TIMMS \& PIRLS. (2018, July 24). Retrieved from https:/ / timssandpirls.bc.edu

Turs,ucu, S., Spandaw, J., \& de Vries M. J. (2018b). Search for symbol sense behavior: students in upper secondary education solving algebraic physics problems. Research in Science Education, 48(5), 1-27. https:/ / doi.org/10.1007/s11165-018-9766-Z

Turşucu, S., Spandaw, J., Flipse, S., \& de Vries, M. J. (2017). Teachers' beliefs about improving transfer of algebraic skills from mathematics into physics in senior pre-university education. International Journal of Science Education, 39(5), 587-604. https:/ / doi.org/10.1080/09500693.2018.1486520

Turşucu, S., Spandaw, J., Flipse, S., Jongbloed, G., \& de Vries, M. J. (2018a). Teachers' beliefs systems about improving transfer of algebraic skills from mathematics into physics in senior pre-university education. International Journal of Science Education, 40(12), 1-27. https:/ / doi.org/10.1080/09500693.2018.1486520

Van den Heuvel-Panhuizen, M. (2003). The didactical use of models in realistic mathematics education: an example from a longitudinal trajectory on percentage. Educational Studies in Mathematics 54, 9-35. https:// doi.org/10.1023/B:EDUC.0000005212.03219.dc

van Zanten, M., \& van den Heuvel - Panhuizen, M. (2014). Freedom of design: the multiple faces of subtraction in Dutch primary school textbooks. In Y. Li, \& G. Lappan (Eds.), Mathematics curriculum in school education (pp. 231-259). New York, NY: Springer. https:/ / doi.org/10.1007/978-94-007-7560-2_12

Walshe, G., Johnston, J., \& McClelland, G. (2017). Integrating mathematics into science: design, development and evaluation of a curriculum model. In Cognitive and Affective Aspects in Science Education Research (pp. 309321). Springer, Cham. https://doi.org/10.1007/978-3-319-58685-4_23

Wenger, R. H. (1987). Cognitive science and algebra learning. In A. Schoenfeld (Ed.), Cognitive science and mathematics education (pp. 217-251). Hillsdale: Lawrence Erlbaum Associates.

Wong, V. J. (2018). The relationship between school science and mathematics education (Doctoral thesis). Retrieved from https://kclpure.kcl.ac.uk/portal/files/95881991/2018_Wong_Victoria_1231276_ethesis.pdf 


\section{APPENDIX}

\section{Physics Teachers' Interview Protocol}

\section{Introduction}

We are greatful that you participated in this interview that will take about 20 minutes and is part of the PhDstudy of the interviewer, $(X)$. We have some questions about the background of your grade-10 physics students, and their mathematics and physics textbooks.

\section{Interview aim}

The purpose of this interview is to select three grade-10 physics students who have a sufficient mathematics grade $(\geq 5.5)$ and an insufficient physics grade. Later, during interviews they will solve algebraic physics problems while being videotaped and thinking aloud (Charters, 2003). These interviews may offer deeper understanding about how students' application of algebraic skills from mathematics in physics can be improved.

\section{Interview strategy}

Could you please answer the questions of the part 'Questions about background of students' below? We note that the students' names will be anonymized. Would you consent to your students who are engaged in this study?

\section{Questions about background of students}

1. Could you please offer us the grade-10 students' names having a sufficient mathematics grade $(\geq 5.5)$ and an insufficient physics grade?

2. Could you please offer us insight in their attitude towards learning?

3. Could you please tell us which physics textbook they use, and why this textbook was chosen?

4. Could you please tell us which mathematics textbook they use?

\section{The Tasks}

\section{Task 1 specific heat capacity}

When an object is heated, its temperature increases. The formula for this phenomenon is given by: $Q=m \cdot c$. $\Delta T$. Here, $Q$ is the thermal energy in J, $\mathrm{m}$ is the mass in $\mathrm{kg}, c$ is the specific heat capacity and $\Delta T$ is the temperature change in $\mathrm{K}$.

Derive the unit of the specific heat capacity. First, solve for $c$.

\section{Task 2 thermal resistance}

The thermal resistance $R_{\text {therm }}=\frac{d}{\lambda \cdot A}$ is a measure of the thermal conductivity of an object. Here, $d$ is the thickness of the material in $m, A$ is the area of the material in $m^{2}$ and $\lambda$ is the thermal conductivity in $\mathrm{W} \cdot \mathrm{m}^{-1} \cdot \mathrm{K}^{-1}$.

a) Derive the unit of $R_{\text {therm }}$.

The heat flow is given by $P=\frac{\lambda \cdot A \cdot \Delta T}{d}$. It can be shown that the relation between $P$ and $R_{\text {therm }}$ is $P=\frac{\Delta T}{R_{\mathrm{therm}}}$.

b) Show that this is the case.

\section{Task 3 charged particles}

The attractive force between two charged particles is given by $F=f \cdot \frac{q_{1} \cdot q_{2}}{r^{2}}$. Here, $\mathrm{f}$ is a constant, $q_{1}$ and $q_{2}$ are the magnitudes of both charged particles in $C$ and $r$ the distance between the centers of the particles in $m$.

Derive the unit of the constant $f$. First, solve for $f$. 


\section{Systematic Solution Set}

\section{Task 1 specific heat capacity}

Procedure 1 (LS): division of both sides of $Q=m \cdot[c] \cdot \Delta T$ with $m$; result:

$\frac{Q}{m}=[c] \cdot \Delta T$. Procedure $2(\mathrm{LS})$ : division of both sides of $\frac{Q}{m}=[c] \cdot \Delta T$ with $\Delta T$; result: $[c]=\frac{Q}{m \cdot \Delta T}$. Procedure 3 (BAS): substitution. Each quantity in the formula is replaced with its corresponding unit, except for $[c]$; result: $[c]=\frac{\mathrm{J}}{\mathrm{kg} \cdot \mathrm{K}}$. The solution $[c]=\mathrm{J} \cdot \mathrm{kg}^{-1} \cdot \mathrm{K}^{-1}$ was also regarded as correct.

\section{Task 2 thermal resistance}

a) Procedure 1 (BAS): substitution. Each quantity in the formula is replaced with its corresponding unit, except for $\left[R_{\text {therm }}\right]$; result: $\left[R_{\text {therm }}\right]=\frac{\mathrm{m}}{\mathrm{W} \cdot \mathrm{m}^{-1} \cdot \mathrm{K}^{-1} \cdot \mathrm{m}^{2}}$. Procedure $2(\mathrm{BAS}):\left[R_{\text {therm }}\right]=\mathrm{m}^{+2-2} \cdot \mathrm{W}^{-1} \cdot \mathrm{K}$; result: $\left[R_{\text {therm }}\right]=\mathrm{W}^{-1}$. $\mathrm{K}$.

b) Procedure 1 (PS): inverting both sides of $R_{\text {therm }}=\frac{d}{\lambda \cdot A}$; result: $\frac{1}{R_{\text {therm }}}=\frac{\lambda \cdot A}{d}$.

Procedure 2 (PS): substitution. Replace $\frac{\lambda \cdot A}{d}$ in $P=\frac{\lambda \cdot A \cdot \Delta T}{d}$ by $\frac{1}{R_{\text {therm }}}$; result: $P=\frac{\Delta T}{R_{\text {therm }}}$.

\section{Task 3 charged particles}

Procedure 1 (LS): multiplication of both sides of $F_{C}=\frac{f \cdot Q \cdot q}{r^{2}}$ with $r^{2}$; result: $r^{2} \cdot F_{C}=f \cdot Q \cdot q$. Procedure 2 (LS): division of both sides with $q$; result: $\frac{r^{2} \cdot F_{C}}{q}=f \cdot Q$. Procedure 3 (LS): division of both sides with $Q$; result: $\frac{r^{2} \cdot F_{C}}{Q \cdot q}=f$. Procedure 4 (BAS): substitution. Each quantity in the formula is replaced with its corresponding unit, except for $[f]$; result: $[f]=\frac{\mathrm{m}^{2} \cdot \mathrm{N}}{C^{2}}$. The solution $[c]=\mathrm{m}^{2} \cdot \mathrm{N} \cdot \mathrm{C}^{-2}$ was also regarded as correct.

\section{Shift Problems}

\section{Exemplary task}

The force of gravity at the earth's surface on an object is given by $F_{g}=m \cdot g$. Here, $m$ is the mass in $\mathrm{kg}$ and $g$ its acceleration due to gravity in $\frac{\mathrm{m}}{\mathrm{s}^{2}}$.

a) Solve for $m$

'Activation hint'. In mathematics class you have learned that

when $y=\boldsymbol{a} \cdot b$ and you want to solve for $\boldsymbol{a}=\cdots$, then the $b$ on the right side should be 'taken away'. This can be done by dividing both sides of the equals sign through $b$. So, $\frac{y}{b}=\frac{\boldsymbol{a} \cdot \boldsymbol{b}}{b}$ and because $\frac{b}{b}=1$, this yields $\boldsymbol{a}=\frac{y}{b}$.

Application of the 'activation hint' yields $F_{g}=m \cdot g, \frac{F_{g}}{g}=\frac{m \cdot g}{g}, m=\frac{F_{g}}{g}$.

b) Find the unit of $F_{g}$

'Strategic hint'. First use the unit-operator [] and replace $F_{g}$ by $\left[F_{g}\right]$, and the other quantities by their units.

Application of the 'strategic hint' yields $\left[F_{g}\right]=\mathrm{kg} \cdot \frac{\mathrm{m}}{\mathrm{s}^{2}}$.

Now you have seen how the 'activation hint' and the 'strategic hint' are applied, I ask you to apply them in the tasks below. Please follow the 'activation' and 'strategic' hints in subsequent order.

\section{Task 1 specific heat capacity (see subsection 'The Tasks')}

Derive the unit of the specific heat capacity. First, solve for $c$.

(1). 'Strategic hint 1': first solve for $c$.

(2). 'Activation hint': in mathematics class you have learned that...

...when $y=\boldsymbol{a} \cdot b$ and you want to solve for $\boldsymbol{a}=\cdots$, then the $b$ on the right side should be 'taken away'. This can be done by dividing both sides of the equals sign through $b$. So, $\frac{y}{b}=\frac{a \cdot b}{b}$ and because $\frac{b}{b}=1$, this yields $\boldsymbol{a}=\frac{y}{b}$.

(3). 'Strategic hint 2': use the unit-operator []. 


\section{Task 2 thermal resistance (see subsection 'The Tasks')}

a) Derive the unit of $R_{\text {thermal }}$.

(1). 'Strategic hint'. First use the unit-operator [].

(2). 'Activation hint'. In mathematics class you have learned that...

...when $y=\frac{a}{\frac{c}{d}}$, this can be simplified into a simpler expression by multiplying both sides of the equals sign by $\frac{d}{d}=1$ giving $y=\frac{a}{\frac{c}{d}} \cdot \frac{d}{d}$. Now, the denominator becomes $\frac{c}{d} \cdot d$. Since, $\frac{d}{d}=1$, the denominator equals $c$. Finally, we write $y=\frac{a d}{c}$.

The heat flow is given by $P=\frac{\lambda \cdot A \cdot \Delta T}{d}$. It can be shown that the relation between $P$ and $R_{\text {thermal }}$ is $P=\frac{\Delta T}{R_{\text {thermal }}}$.

b) Show that this is the case.

(1). 'Strategic hint'. Rewrite $R_{\text {therm }}$ into $1 / R_{\text {therm }}$.

(2). 'Activation hint'. In mathematics class you have learned that...

...when $y=a b$ and $z=a b$ then the following relation apply: $y=a b=z$. This means that $y=z$.

\section{Task 3 charged particles (see subsection 'The Tasks')}

Derive the unit of the constant $f$. First, solve for $f$.

(1). 'Strategic hint'. First solve for $f$.

(2). 'Activation hint'. In mathematics class you have learned that...

...when $y=\frac{a}{b}$ and you want to solve for $a=\cdots$, then the $\frac{1}{b}$ on the right side should be 'taken away'. This can be done by multiplying both sides of the equals sign by $b$. So, $y \cdot b=\frac{a}{b} \cdot b$ and because $\frac{b}{b}=1$, this yields $a=y b$.

(3). 'Strategic hint'. Use the unit-operator [].

\section{Physics Students' Interview Protocol}

\section{Introduction}

We are greatful that you participated in this interview that consists of two parts. In the first section we ask you some questions about your background. The second section is concerned with algebraic problem-solving in physics where we ask you to solve four tasks while thinking aloud and being videotaped.

\section{First section: questions about your background}

1. Could you please tell me why you chose mathematics and science subjects?

2. Could you please tell me your ideas about mathematics and physics?

3. Could you please offer me your grades for mathematics and physics?

\section{Second section: algebraic problem-solving in physics}

Could you please solve the three tasks 'Task 1: specific heat capacity', 'Task 2: thermal resistance' and 'Task 3: charged particles' below while thinking aloud. Could you please write down as many intermediate steps as possible. You will only be interrupted if it remains silent for approximately one minute, or a procedure or reasoning is not clear enough. Your name will be anonymized, and all information that you offered during this interview will only be used for a PhD-research.

\section{http://www.ejmste.com}

